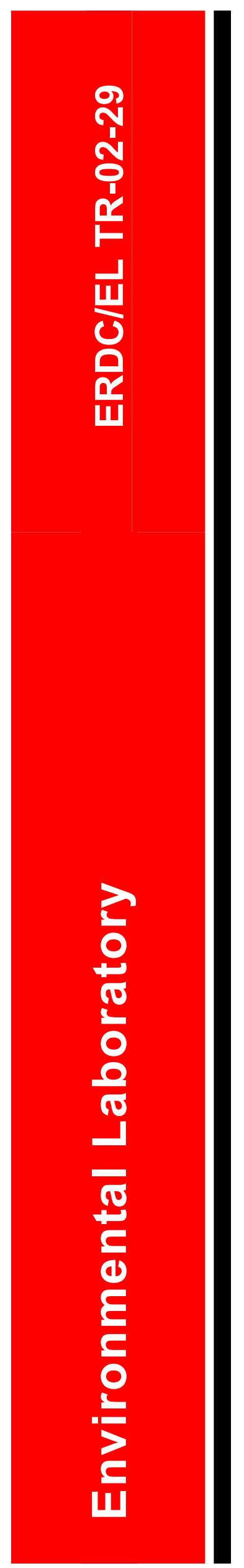

\title{
User's Manual for NAVEFF Navigation Effects Model
}

Scott Bourne

September 2002

Approved for public release; distribution is unlimited. 
The contents of this report are not to be used for advertising, publication, or promotional purposes. Citation of trade names does not constitute an official endorsement or approval of the use of such commercial products.

The findings of this report are not to be construed as an official Department of the Army position, unless so designated by other authorized documents. 


\section{User's Manual for NAVEFF Navigation Effects Model}

by Scott Bourne

Environmental Laboratory

U.S. Army Engineer Research and Development Center 3909 Halls Ferry Road

Vicksburg, MS 39180-6199

Final report

Approved for public release; distribution is unlimited 


\section{Contents}

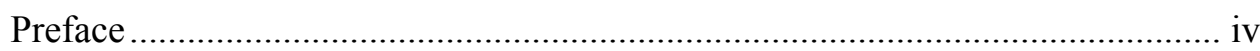

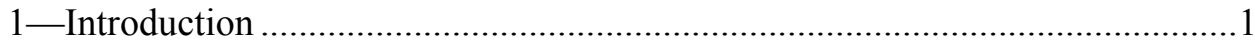

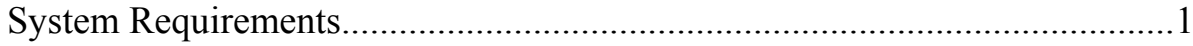

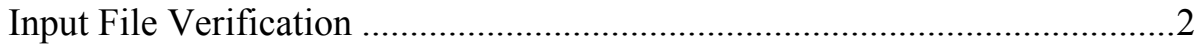

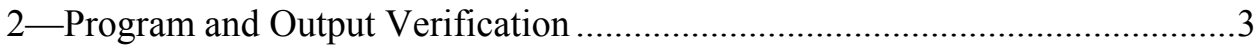

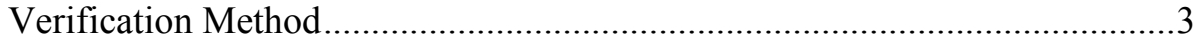

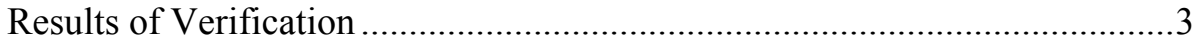

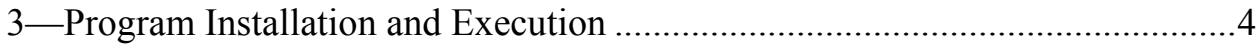

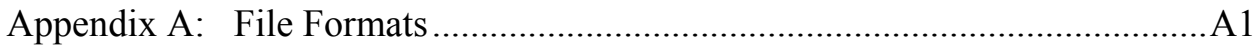

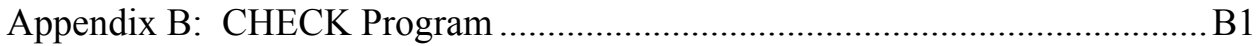

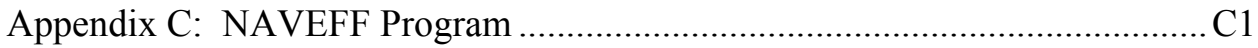

SF 298 


\section{Preface}

This report was prepared for the Upper Mississippi River Navigation Study (UMRS) sponsored by the U.S. Army Engineer District, Rock Island. The user guide was written by Scott Bourne, Environmental Laboratory (EL), U.S. Army Engineer Research and Development Center (ERDC), Vicksburg, MS. Dr. Steve Maynord, Coastal and Hydraulics Laboratory (CHL), ERDC, provided technical review. Project manager for the UMRS was Dr. Kenneth Barr, Rock Island District.

This work was conducted under the direction of Dr. David J. Tazik, Chief, Ecosystem Evaluation and Engineering Division, EL, and Mr. Harold W. West, Chief, Environmental Systems Branch. Director of EL was Dr. Edwin A. Theriot.

At the time of publication of this report, Dr. James R. Houston was Director of ERDC, and COL John W. Morris III, EN, was Commander and Executive Director.

This report should be cited as follows:

Bourne, S. (2002). “User's manual for NAVEFF navigation effects model," ERDC/EL TR-02-29, U.S. Army Engineer Research and Development Center, Vicksburg, MS.

The contents of this report are not to be used for advertising, publication, or promotional purposes. Citation of trade names does not constitute an official endorsement or approval of the use of such commercial products. 


\section{Introduction}

The model for Navigation Effects (NAVEFF) models physical forces of tow boat passage across a river cross section. This is a one-dimensional model containing empirical relations for estimating physical forces. ${ }^{1}$

The physical forces that are modeled in NAVEFF will be expressed as:

a. Secondary wave height.

b. Scour.

c. Drawdown.

d. Velocity change.

e. Shear stress.

Physical forces that are generated by NAVEFF are based on 108 boat types. These 108 boat types are a combination of three boat speeds, three sail line positions, three water stages, direction (upstream or downstream), propeller type (open wheel or kort nozzle), boat size, and draft. The fleet characteristics are described in Appendix A (filename P\#_PARM.DAT).

\section{System Requirements}

These computer programs have been tested and run on both UNIX and Windows. A comparison of a UNIX run and a Windows run showed that the output was identical. Both the CHECK and NAVEFF programs were compiled on both systems using Visual Fortran.

Due to the size of the output files, a large amount of hard disk space will generally be required. Typically 1 to 3 GB of free disk space will be needed. Pools 4, 8, 13, 26, and Lagrange will require more disk space since the program will be executed at each half river mile.

\footnotetext{
${ }^{1}$ Maynord, S., Bourne, S., Graves, M., Landwehr, K., and Knight, S., "UMR-IWW System Models Report - Physical Effects Model," U.S. Army Engineer District, Rock Island, Rock Island, IL, in preparation.
} 


\section{Input File Verification}

NAVEFF requires six input files. A CHECK program (CHECK.EXE), developed to test these input files, verifies that the input files are in the proper format. The check program looks at each sail line position and ascertains if the cell selected for the sail line and the two adjacent cells are deep enough for a tow with a $9-\mathrm{ft}(2.743-\mathrm{m})$ draft to pass. The program checks the cells that are within the channel extents (extents defined in P\#_chan.dat input file, Coastal and Hydraulics Laboratory (CHL), U.S. Army Engineer Research and Development Center, Vicksburg, MS) and determines if each of these cells has ambient velocity values and a sediment type. The output from the CHECK program is written to an ASCII file where the results can be viewed. 


\section{Program and Output Verification}

\section{Verification Method}

Verification of the NAVEFF code was done by processing 16 randomly selected traffic configurations in Pool 13 and comparing the NAVEFF output results to an earlier BASIC version of NAVEFF. The NAVEFF output parameters, velocity change, drawdown, scour, shear stress, and secondary wave height, were tabulated and graphically displayed for comparison purposes.

Output verification was done by processing the largest, fastest traffic configuration and the light traffic configuration. Four NAVEFF output parameters (change velocity, drawdown, scour, and shear stress) were selected, and the results were displayed to determine if there was something obviously wrong with the output. The largest, fastest configuration used was the following boat type: upbound direction, fast speed, big boat, loaded barge, kort nozzle, low stage, and middle sailing line. The light boat traffic configuration used was: downbound direction, slow speed, small boat, empty barge, kort nozzle, high stage, and middle sailing line.

\section{Results of Verification}

The comparison of the current NAVEFF code and an earlier BASIC version of the code demonstrated that there were no significant differences in the output generated by both versions.

The output generated by the worst traffic configuration and the light traffic configuration was examined by CHL personnel. Navigation effects values that were generated by these two configurations were determined to fall within an acceptable range of values. 


\section{Program Installation and Execution}

The steps below list the files needed in each directory to execute the CHECK and NAVEFF programs.

\section{Installation}

1. Set up a new directory (pool\#).

2. Copy the NAVEFF and CHECK program executables (.exe) into the new directory.

3. Copy the following input files into the same directory:

p\#_chan.dat p\#_sail.dat

p\#_lmh.dat p\#_elev.dat

p\#_parm.dat p\#_yz.trf

Where: $y=$ stage, $z=$ sailing line

The format of the input files is indicated in Appendix A

\section{Execution of CHECK Program}

4. On the command line of a DOS or UNIX window, type CHECK. The CHECK program code is listed in Appendix B.

5. The user will be prompted for the pool number:

c: $\mid$ pool\# $>>$ check

Enter Pool Number: p\#

6. When the program is finished, the message NORMAL STOP

CONDITIONS will be written to the screen.

7. Two ASCII files will be generated by executing this command, an error file (.err), which will list any errors in the input files, and an output file (.out), which will always be empty.

8. The errors found in the error file refer to the relationship of the p\#_sail.dat and p\#_chan.dat files to the p\#_elev.dat file.

9. Sail line errors are found when the cell selected for the sail line and two adjacent cells have a water depth value of less than $9 \mathrm{ft}(2.743 \mathrm{~m})$. Edit the p\#_sail.dat file to move the sail line position. The left, middle, and right sail line positions are the same for each water stage. When a sail line position is being moved, be sure and move it for each water stage level. 
10. Cells without ambient velocity or sediment type that are within the channel extents set in the $\mathrm{p \#}$ _chan.dat file will produce an error in the error file. These errors are water stage level specific, meaning that the channel extents for the three water stages are different and that error refers to a specific water stage. This error will list all the cells that do not have an ambient velocity value. Edit the p\#_chan.dat and move the channel extent in toward the sail line until all cells have an ambient velocity value.

11. Once all the errors have been corrected, delete the error and output files and re-run the CHECK program.

12. Sometimes moving the channel and sail line will generate more errors, so always re-run the CHECK program after making edits.

13. When the error file comes back empty, the NAVEFF program is ready to be run.

\section{Execution of NAVEFF Program}

14. On the command line of a DOS or UNIX window, type NAVEFF. The NAVEFF program code is listed in Appendix C.

15. The user will be prompted for the traffic file:

c: $\mid$ pool\#I $>$ naveff

Enter Traffic File Name: p\#_yz.trf

Where $\mathrm{y}=$ Stage, $\mathrm{z}=$ sail line

16. NAVEFF will be run nine times for each of the nine traffic files per pool.

17. Each line in the output file contains a unique ID that allows the output data to be incorporated back into the geographic information system database and displayed. 


\section{Appendix A File Formats}

The NAVEFF program requires six input files and generates a single output file. These input and output files are ASCII files and comma delimited. The formats and descriptions of the input files are listed in this section.

\begin{tabular}{|l|l|}
\hline File Name & Description \\
\hline P\#_CHAN.DAT & $\begin{array}{l}\text { This input file contains the bank line position at low, } \\
\text { medium, and high stage for each river mile. }\end{array}$ \\
\hline P\#_ELEV.DAT & $\begin{array}{l}\text { This input file contains the ambient velocities at low, } \\
\text { medium, and high stage. Also, sediment values are } \\
\text { contained in this input file. }\end{array}$ \\
\hline P\#_LMH.DAT & $\begin{array}{l}\text { Low, medium, and high river stage values are contained in } \\
\text { this input file. }\end{array}$ \\
\hline P\#_PARM.DAT & This input file contains the tow boat characteristics. \\
\hline P\#_SAIL.DAT & $\begin{array}{l}\text { Left, middle, and right sail line positions are contained in } \\
\text { this input file. }\end{array}$ \\
\hline P\#_YZ.TRF & $\begin{array}{l}\text { These input files contain the 108 tow boat types for each } \\
\text { river mile. }\end{array}$ \\
\hline P\#_YZ.OUT & NAVEFF output file. \\
\hline
\end{tabular}


P\#_CHAN.DAT

\begin{tabular}{|l|l|l|l|l|}
\hline \multicolumn{5}{|l|}{ Bank Positions } \\
\hline Variable & Description & Type & Format & Example \\
\hline RIVER_MI & River Mile & Numeric & 5.1 & 523.5 \\
\hline LSTG_LBK & $\begin{array}{l}\text { Cell ID Location of Left Bank } \\
\text { Line at Low Stage }\end{array}$ & Character & 10 & 405 L5235 \\
\hline LSTG_RBK & $\begin{array}{l}\text { Cell ID Location of Right } \\
\text { Bank Line at Low Stage }\end{array}$ & Character & 10 & 305 L5235 \\
\hline MSTG_LBK & $\begin{array}{l}\text { Cell ID Location of Left Bank } \\
\text { Line at Medium Stage }\end{array}$ & Character & 10 & 505 L5235 \\
\hline MSTG_RBK & $\begin{array}{l}\text { Cell ID Location of Right } \\
\text { Bank Line at Medium Stage }\end{array}$ & Character & 10 & 605 L5235 \\
\hline HSTG_LBK & $\begin{array}{l}\text { Cell ID Location of Left Bank } \\
\text { Line at High Stage }\end{array}$ & Character & 10 & 905 L5235 \\
\hline HSTG_RBK & $\begin{array}{l}\text { Cell ID Location of Right } \\
\text { Bank Line at High Stage }\end{array}$ & Character & 10 & 805 L5235 \\
\hline
\end{tabular}

\section{Example:}

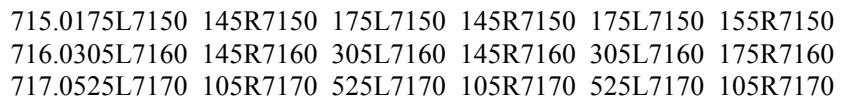


P\#_ELEV.DAT

\begin{tabular}{|c|c|c|c|c|}
\hline \multicolumn{5}{|c|}{ Cell Information Input File } \\
\hline Variable & Description & Type & Format & Example \\
\hline RIVER_MI & River Mile & Numeric & 5.1 & 523.5 \\
\hline CELL_ID & Cell ID & Character & 10 & 305L5235 \\
\hline XCOORD & Mid-Point X Coordinate & Numeric & 12.1 & 713255.2 \\
\hline YCOORD & Mid-Point Y Coordinate & Numeric & 12.1 & 4681465 \\
\hline MSL_M & $\begin{array}{l}\text { Mean Sea Level Elevation at } \\
\text { Flat Pool (M) }\end{array}$ & Numeric & 8.3 & 179.821 \\
\hline AMBVEL_L & $\begin{array}{l}\text { Ambient Current Velocity at } \\
\text { Low Stage }(\mathrm{M} / \mathrm{S})\end{array}$ & Numeric & 8.3 & 0.141 \\
\hline AMBVEL_M & $\begin{array}{l}\text { Ambient Current Velocity at } \\
\text { Medium Stage }(\mathrm{M} / \mathrm{S})\end{array}$ & Numeric & 8.3 & 0.291 \\
\hline AMBVEL_H & $\begin{array}{l}\text { Ambient Current Velocity at } \\
\text { High Stage }(\mathrm{M} / \mathrm{S})\end{array}$ & Numeric & 8.3 & 0.346 \\
\hline D50_GSZ_MM & $\begin{array}{l}\text { D50 Particle Grain Size } \\
\text { (MM) }\end{array}$ & Numeric & 8.3 & 0.081 \\
\hline D50_VEL_CMS & $\begin{array}{l}\text { D50 Particle Fall Velocity } \\
(\mathrm{CM} / \mathrm{S})\end{array}$ & Numeric & 8.3 & 0.531 \\
\hline COH_SED & Cohesive Sediment & Numeric & 6 & 2 \\
\hline COH_CLASS & Cohesive Class for Group 2 & Numeric & 6 & 2 \\
\hline
\end{tabular}

\section{Example:}

715.0175L7150 624274.7 4873457.0 194.706 $0.0740 .232 \quad 0.6480 .6548 .35630$ 715.0165L7150 624270.9 4873447.5 194.554 0.077 0.244 0.6760 .6548 .35630

715.0155L7150 624267.2 4873438.5 193.091 $0.106 \quad 0.3430 .9120 .6548 .35630$ 
P\#_LMH.DAT

\begin{tabular}{|l|l|l|l|l|}
\hline Water Stages at Each River Mile \\
\hline Variable & Description & Type & Format & Example \\
\hline RIVER_MI & River Mile & Numeric & 5.1 & 523.5 \\
\hline LSTG_MSL_M & Low Stage Value in MSL (M) & Numeric & 8.2 & 177.49 \\
\hline MSTG_MSL_M & $\begin{array}{l}\text { Medium Stage Value in } \\
\text { MSL (M) }\end{array}$ & Numeric & 8.2 & 177.76 \\
\hline HSTG_MSL_M & High Stage Value & Numeric & 8.2 & 177.88 \\
\hline
\end{tabular}

Example:

$\begin{array}{lllll}715.0 & 196.69 & 196.47 & 196.81\end{array}$

$\begin{array}{lllll}716.0 & 196.69 & 196.50 & 196.96\end{array}$

$\begin{array}{lllll}717.0 & 196.69 & 196.54 & 197.11\end{array}$ 


\section{P\#_PARM.DAT}

\begin{tabular}{|l|l|l|l|}
\hline \multicolumn{5}{|l|}{ Boat Characteristics } & (Position 9) & Remark \\
\hline (Position 1) & (Position 2) & & \\
\hline$\#$ & Speed & & Slow speed (M/S) \\
\hline S & 2.24 & & Medium speed (M/S) \\
\hline M & 2.91 & & Fast speed (M/S) \\
\hline F & 3.58 & & \\
\hline Blank Row) & & & \\
\hline$\#$ & Size & $(1$ Blank) 45.72 & Light size (M) \\
\hline L & 10.67 & 178.31 & Small size (M) \\
\hline S & 10.67 & 237.74 & Medium size (M) \\
\hline M & 21.34 & 297.18 & Big size (M) \\
\hline B & 32.00 & & \\
\hline Blank Row) & & & \\
\hline$\#$ & Draft & & Loaded (M) \\
\hline L & 2.74 & & Mixed (M) \\
\hline M & 2.13 & & Empty (M) \\
\hline E & 0.61 & & \\
\hline
\end{tabular}

\section{Example:}

\# SPEED
S 2.24
M 2.91
F 3.58
\# SIZE
L 10.6745 .72
S 10.67178 .31
M 21.34237 .74
B 32.00297 .18
\# DRAFT
L 2.74
M 2.13
E 0.61


P\#_SAIL.DAT

\begin{tabular}{|c|c|c|c|c|}
\hline \multicolumn{5}{|c|}{ Sail Line Position } \\
\hline \begin{tabular}{|l|} 
Variable \\
\end{tabular} & Description & Type & Format & Example \\
\hline RIVER_MI & River Mile & Numeric & 5.1 & 523.5 \\
\hline LSTG_LSL & $\begin{array}{l}\text { Cell ID Location of Left } \\
\text { Sailing Line at Low Stage }\end{array}$ & Character & 10 & 305L5235 \\
\hline LSTG_MSL & $\begin{array}{l}\text { Cell ID Location of Middle } \\
\text { Sailing Line at Low Stage }\end{array}$ & Character & 10 & 55L5235 \\
\hline LSTG_RSL & $\begin{array}{l}\text { Cell ID Location of Right } \\
\text { Sailing Line at Low Stage }\end{array}$ & Character & 10 & 205R5235 \\
\hline MSTG_LSL & $\begin{array}{l}\text { Cell ID Location of Left } \\
\text { Sailing Line at Medium Stage }\end{array}$ & Character & 10 & 305L5235 \\
\hline MSTG_MSL & $\begin{array}{l}\text { Cell ID Location of Middle } \\
\text { Sailing Line at Medium Stage }\end{array}$ & Character & 10 & 55L5235 \\
\hline MSTG_RSL & $\begin{array}{l}\text { Cell ID Location of Right } \\
\text { Sailing Line at Medium Stage }\end{array}$ & Character & 10 & 205R5235 \\
\hline HSTG_LSL & $\begin{array}{l}\text { Cell ID Location of Left } \\
\text { Sailing Line at High Stage }\end{array}$ & Character & 10 & 305L5235 \\
\hline HSTG_MSL & $\begin{array}{l}\text { Cell ID Location of Middle } \\
\text { Sailing Line at High Stage }\end{array}$ & Character & 10 & 55L5235 \\
\hline HSTG_RSL & $\begin{array}{l}\text { Cell ID Location of Right } \\
\text { Sailing Line at High Stage }\end{array}$ & Character & 10 & 205R5235 \\
\hline
\end{tabular}

\section{Example:}

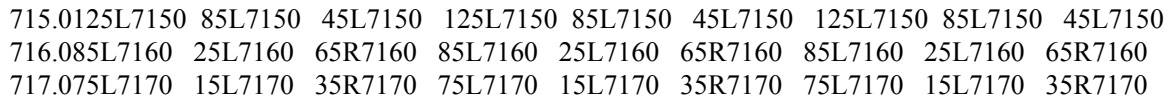


P\#_YZ.TRF

\begin{tabular}{|l|l|l|l|l|}
\hline \multicolumn{5}{|l|}{ Traffic Input File } \\
\hline Variable & Description & Type & Format & Example \\
\hline RIVER_MI & River Mile & Numeric & 5.1 & 523.5 \\
\hline DIR & Upbound/Downbound & Character & 1 & $\mathrm{U}$ \\
\hline SPEED & Fast/Medium/Slow & Character & 1 & $\mathrm{~F}$ \\
\hline SIZE & Big/Medium/Slow & Character & 1 & $\mathrm{M}$ \\
\hline DRAFT & Load/Mixed/Empty & Character & 1 & E \\
\hline TURBINE & Open Wheel/Kort Nozzle & Character & 1 & $\mathrm{O}$ \\
\hline STAGE & High/Medium/Low & Character & 1 & $\mathrm{~L}$ \\
\hline SL_POS & Left/Middle/Right & Character & 1 & $\mathrm{R}$ \\
\hline
\end{tabular}

Example:

715.0,U,S,S,E,K,H,M

716.0,U,S,S,E,O,H,M

717.0,U,S,S,M,K,H,M 
P\#_YZ.OUT

\begin{tabular}{|c|c|c|c|c|}
\hline \multicolumn{5}{|c|}{ NAVEFF Output Variables } \\
\hline Variable & Description & Type & Format & Example \\
\hline RIVER_MI & River Mile & Numeric & 5.1 & 523.5 \\
\hline DIR & Upbound/Downbound & Character & 1 & $\mathrm{U}$ \\
\hline SPEED & Fast/Medium/Slow & Character & 1 & $\mathrm{~F}$ \\
\hline SIZE & Big/Medium/Small & Character & 1 & B \\
\hline DRAFT & Load/Mixed/Empty & Character & 1 & $\mathrm{~L}$ \\
\hline TURBINE & Open Wheel/Kort Nozzle & Character & 1 & $\mathrm{O}$ \\
\hline STAGE & High/Medium/Low & Character & 1 & $\mathrm{H}$ \\
\hline SL POS & Left/Middle/Right & Character & 1 & $\mathrm{~L}$ \\
\hline TRAFFIC & $\begin{array}{l}\text { Variables } 2 \text { Thru } 6 \\
\text { Concatenated }\end{array}$ & Character & 5 & UFBLO \\
\hline STG_SL & Variables 7 and 8 Con & Character & 2 & HL \\
\hline CELL_ID & Cell ID & Character & 10 & $\begin{array}{l}305 \mathrm{~L} 523 \\
5\end{array}$ \\
\hline DEPTH & Depth (M) & Numeric & 6.2 & 3.55 \\
\hline VEL_CHANGE & $\begin{array}{l}\text { Maximum Velocity Change } \\
(\mathrm{M} / \mathrm{S})\end{array}$ & Numeric & 7.3 & 0.205 \\
\hline DRAWDOWN & Drawdown $(\mathrm{M})$ & Numeric & 7.3 & 0.107 \\
\hline SL_DIST & Distance from Sailing Line (M) & Numeric & 7.1 & 20.8 \\
\hline SEC_WH & Secondary Wave Height (M) & Numeric & 7.3 & 0.107 \\
\hline MX_SCOUR & Maximum Scour (M) & Numeric & 9.4 & -0.1816 \\
\hline MX_SHEAR & Maximum Shear Stress (PA) & Numeric & 9.4 & 176.2432 \\
\hline AMB_FLUX & $\begin{array}{l}\text { Ambient Flux (Particle/Particle } \\
\text { By Vol.) }\end{array}$ & Numeric & 12.7 & $\begin{array}{l}0.000111 \\
2\end{array}$ \\
\hline MX_AFLUX & $\begin{array}{l}\text { Maximum Ambient Flux } \\
\text { (Particle/Particle By Vol.) }\end{array}$ & Numeric & 12.7 & $\begin{array}{l}0.284812 \\
3\end{array}$ \\
\hline AMB_SHEAR & Ambient Shear Stress (PA) & Numeric & 9.4 & 176.2432 \\
\hline
\end{tabular}

\section{Example:}

328.0,D,M,S,E,O,H,L,DMSEO,HL,155L3280,3.35,0.006,0.002, -92.4,0.093,0.0000,0.8766, $0.000000,0.000000,0.8766$

328.0,D,M,S,E,O,H,L,DMSEO,HL, 145L3280,4.85,0.007,0.002,-82.3,0.097,-0.0001,1.2370, $0.000007,0.000007,1.2370$

328.0,D,M,S,E,O,H,L,DMSEO,HL,135L3280,6.05,0.008,0.003,-71.7,0.102,-0.0001,1.5536, $0.000012,0.000012,1.5536$

328.0,D,M,S,E,O,H,L,DMSEO,HL,125L3280,5.75,0.010,0.003,-61.5,0.107,-0.0001,1.4788, $0.000011,0.000011,1.4788$ 


\title{
Appendix B CHECK Program
}

Files

\author{
Input \\ P\# chan.dat \\ $\mathrm{P} \#$ elev.dat \\ P\# lmh.dat \\ P\#_parm.dat \\ P\#_sail.dat \\ P\#_yz.trf \\ where: \# = Pool Number \\ $\mathrm{y}=$ Stage, $\mathrm{z}=$ Sailing Line
}




\section{Source Code}

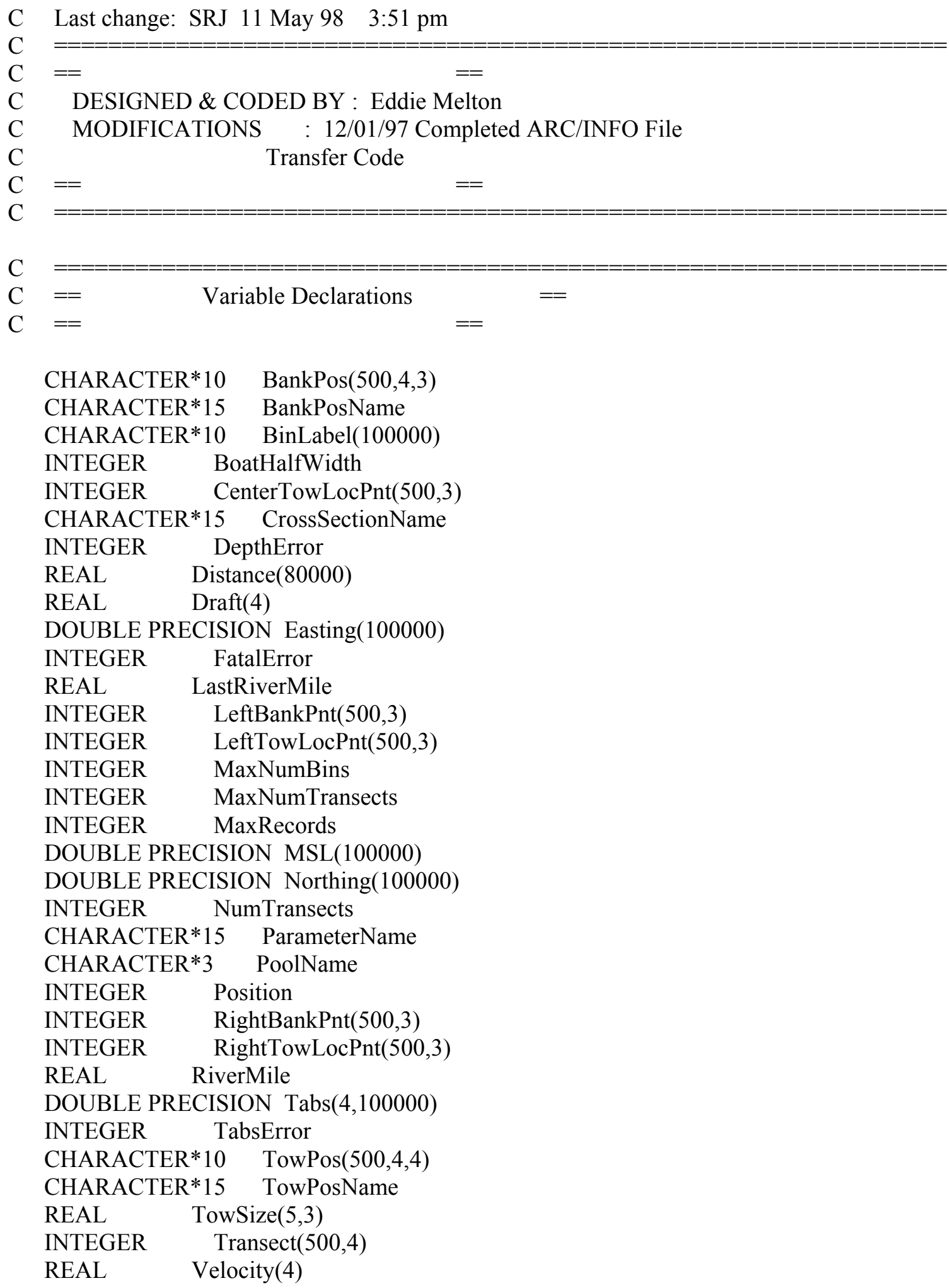


DOUBLE PRECISION WaterDepth $(4,100000)$

REAL WaterLevel $(500,4)$

CHARACTER*1 WaterLevelId(3)

CHARACTER *15 WaterLevelName

REAL Width(80000)

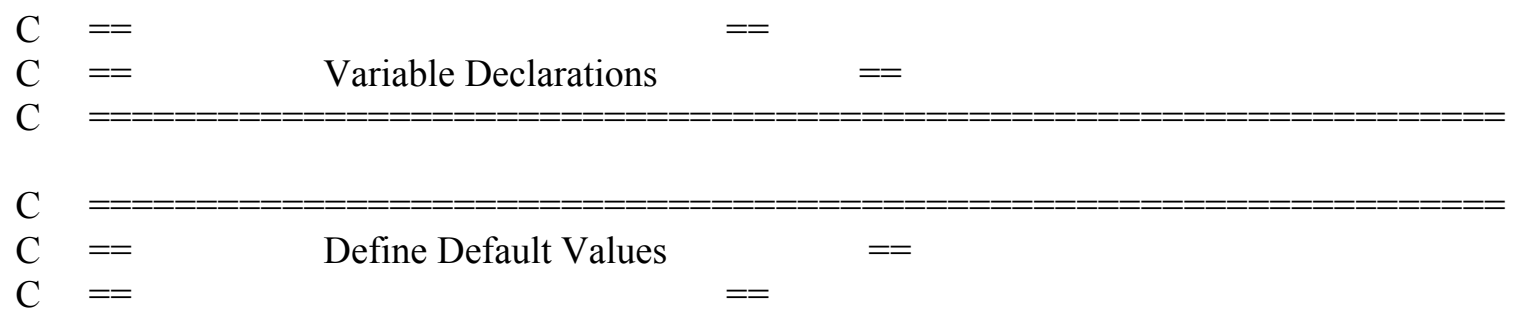

DATA MaxNumTransects / 100 /

DATA MaxNumBins / 800 /

MaxRecords $=$ MaxNumTransects $*$ MaxNumBins

NumTransects $=$ MaxNumTransects

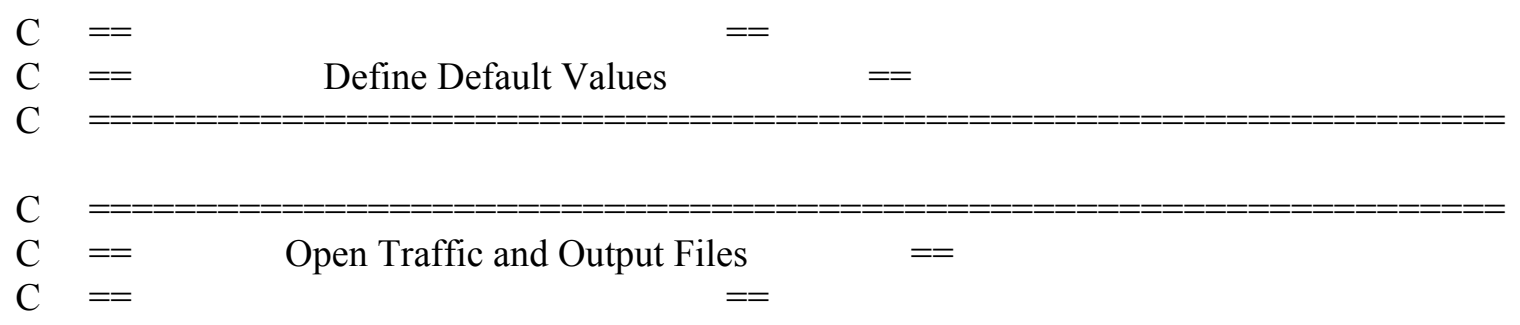

WRITE $(*, *)$ 'Enter Pool Name: '

$\operatorname{READ}(*, *)$ PoolName

$\operatorname{OPEN}(8$, FILE $=$ PoolName//'.err', STATUS='replace')

$\operatorname{OPEN}(9$, FILE=PoolName//'.out', STATUS='replace')

$\begin{array}{lll}\mathrm{C}== & = & \\ \mathrm{C}== & \text { Open Traffic and Output Files } & == \\ \mathrm{C}=========================================================\end{array}$

FatalError $=0$

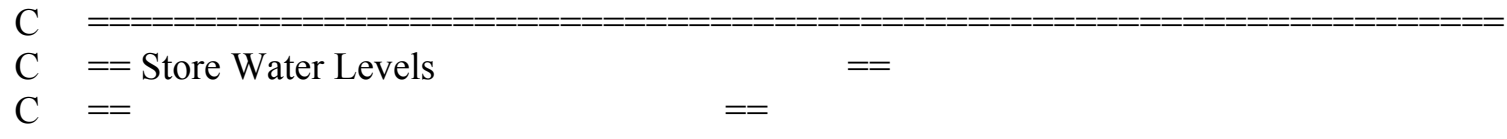

WaterLevelName $=$ PoolName//'_lmh.dat'

$\operatorname{OPEN}(2$, FILE $=$ WaterLevelName, STATUS='old')

DO $\mathrm{i}=1$, NumTransects

$\operatorname{READ}(2, *, E N D=200)$ RiverMile, WaterLevel(i,1),

* WaterLevel(i,2), WaterLevel(i,3) 


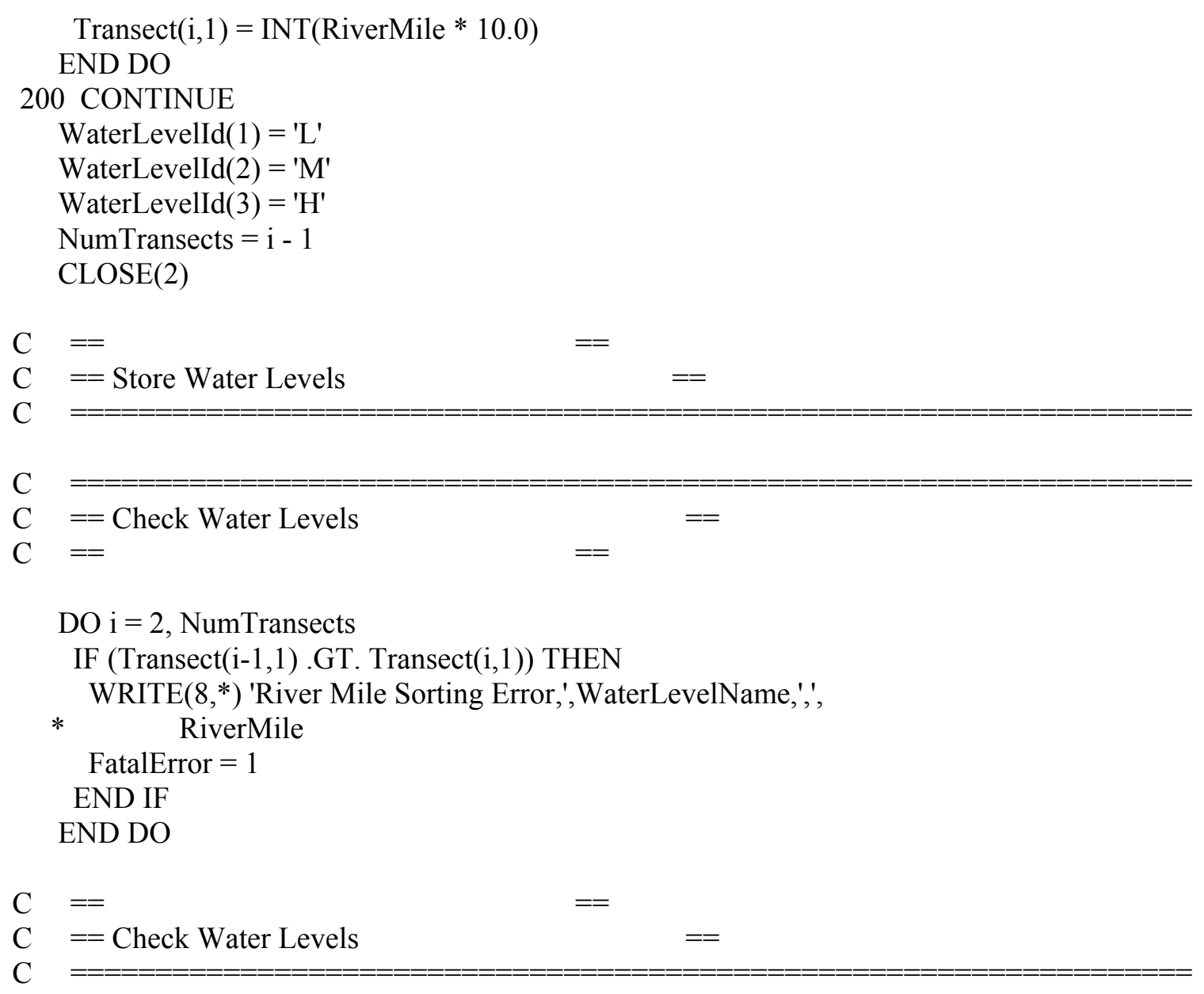

IF (FatalError .EQ. 1) GOTO 999

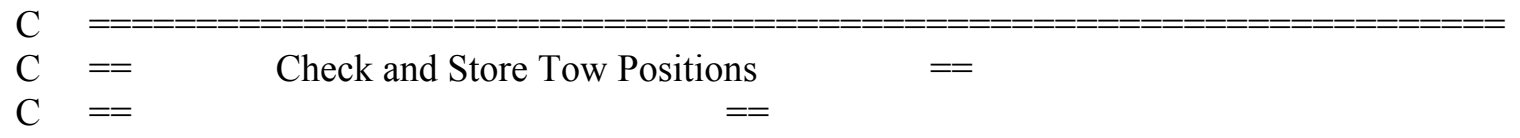

TowPosName $=$ PoolName//'_sail.dat' $\operatorname{OPEN}(3$, FILE $=$ TowPosName, STATUS $=$ 'old')

DO $\mathrm{i}=1$, NumTransects

$\operatorname{READ}(3,350, \mathrm{END}=300)$ RiverMile,

* TowPos(i,1,1),TowPos(i,1,2),TowPos(i,1,3),

* TowPos(i,2,1),TowPos(i,2,2),TowPos(i,2,3),

* TowPos(i,3,1),TowPos(i,3,2),TowPos(i,3,3)

IF(Transect(i,1) .NE. INT(RiverMile * 10.0)) THEN

WRITE $(8, *)$ 'River Mile Sorting Error,',TowPosName,',',

* RiverMile

FatalError $=1$

ENDIF

END DO

300 CONTINUE

CLOSE(3) 
350 FORMAT(f5.1, a10, a10, a10, a10, a10, a10, a10, a10, a10)

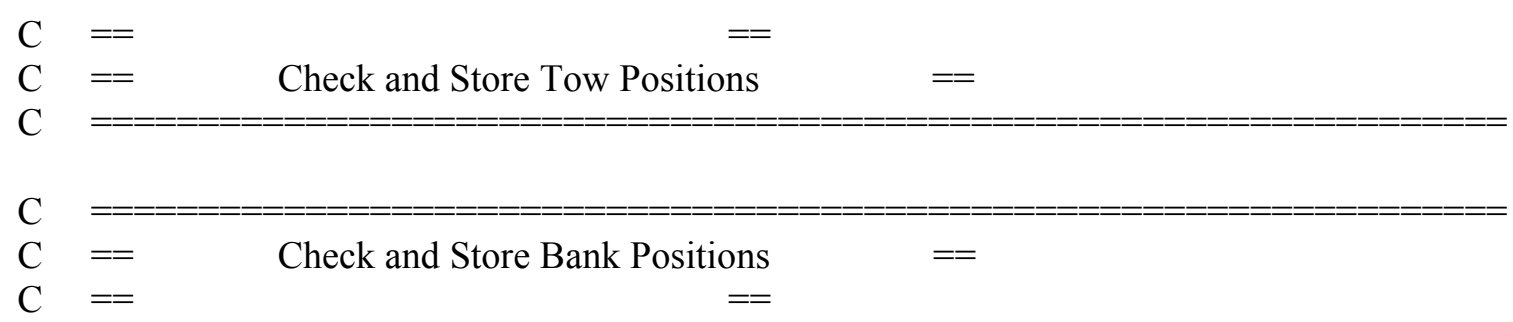

BankPosName $=$ PoolName $/ /$ ' chan.dat'

OPEN(4, FILE=BankPosName, STATUS='old')

DO $i=1$, NumTransects

$\operatorname{READ}(4,450, \mathrm{END}=400)$ RiverMile,

* BankPos(i,1,1), BankPos(i,1,2),

* $\quad \operatorname{BankPos}(i, 2,1), \operatorname{BankPos}(i, 2,2)$,

* BankPos(i,3,1), BankPos(i,3,2)

IF(Transect(i,1) .NE. INT(RiverMile * 10.0)) THEN

WRITE $\left(8,{ }^{*}\right)$ 'River Mile Sorting Error,',BankPosName,',',

* RiverMile

FatalError $=1$

ENDIF

END DO

400 CONTINUE

CLOSE(4)

450 FORMAT(f5.1, a10, a10, a10, a10, a10, a10)

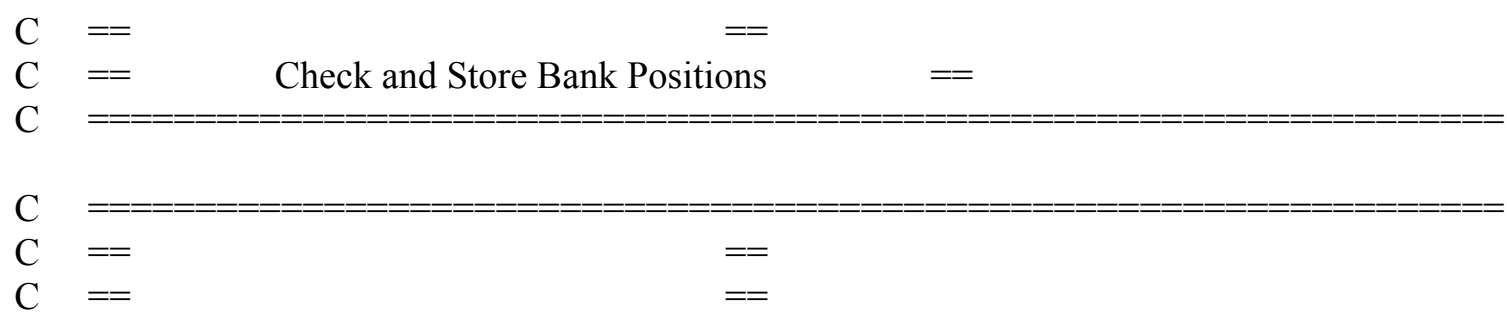

ParameterName $=$ PoolName $/ /$ ' parm.dat'

OPEN $(5$, FILE $=$ ParameterName, STATUS $=$ 'old')

$\operatorname{READ}(5, *)$

DO $\mathrm{i}=1,3$

$\operatorname{READ}(5,550)$ Velocity(i)

ENDDO

$\operatorname{READ}(5, *)$

$\operatorname{READ}(5, *)$

DO $\mathrm{i}=1,4$

$\operatorname{READ}(5,551)$ TowSize $(i, 1)$, TowSize $(i, 2)$

ENDDO

$\operatorname{READ}(5, *)$

$\operatorname{READ}(5, *)$

DO $\mathrm{i}=1,3$

$\operatorname{READ}(5,552) \operatorname{Draft}(\mathrm{i})$ 
ENDDO

CLOSE(5)

550 FORMAT(2x, f4.2)

551 FORMAT(2x, f5.2, $\mathrm{x}, \mathrm{f6.2})$

552 FORMAT $(2 \mathrm{x}, \mathrm{f} 4.2)$

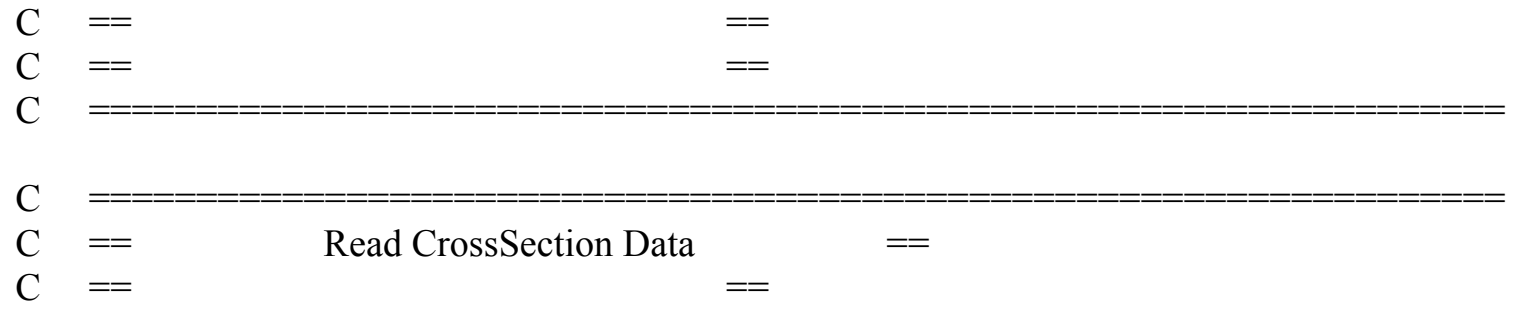

CrossSectionName $=$ PoolName $/ /$ ' elev.dat'

OPEN $(6$, FILE $=$ CrossSectionName, STATUS='old')

$\mathrm{j}=0$

LastRiverMile $=0.0$

DO $\mathrm{i}=1$, MaxRecords

$\operatorname{READ}(6,650, \mathrm{END}=600)$ RiverMile, BinLabel(i),

$*$

Easting(i), Northing(i)

* $\quad \operatorname{MSL}(\mathrm{i}), \operatorname{Tabs}(1, \mathrm{i}), \operatorname{Tabs}(2, \mathrm{i}), \operatorname{Tabs}(3, \mathrm{i})$

IF(ABS(RiverMile - LastRiverMile) .LT. 0.01) THEN

Transect $(\mathrm{j}, 3)=\mathrm{i}$

IF(Transect $(j, 1)$.NE. INT(RiverMile * 10.0)) THEN

WRITE $\left(8,{ }^{*}\right)$ 'River Mile Sorting Error,',CrossSectionName,',',

* RiverMile

FatalError $=1$

ENDIF

ELSE

$\mathrm{j}=\mathrm{j}+1$

Transect $(\mathrm{j}, 2)=\mathrm{i}$

LastRiverMile $=$ RiverMile

IF(Transect $(\mathrm{j}, 1)$.NE. INT(RiverMile * 10.0)) THEN

WRITE $\left(8,{ }^{*}\right)$ 'River Mile Sorting Error,',CrossSectionName,',',

* RiverMile

FatalError $=1$

ENDIF

ENDIF

ENDDO

600 CONTINUE

MaxRecords $=\mathrm{i}-1$

CLOSE(6)

650 FORMAT(f5.1, a10, f12.1, f12.1, f8.1, f8.1, f8.1, f8.1)

$\mathrm{C}==$

$\mathrm{C}==$

$\mathrm{C}$

Read CrossSection Data = 
IF (FatalError .EQ. 1) GOTO 999

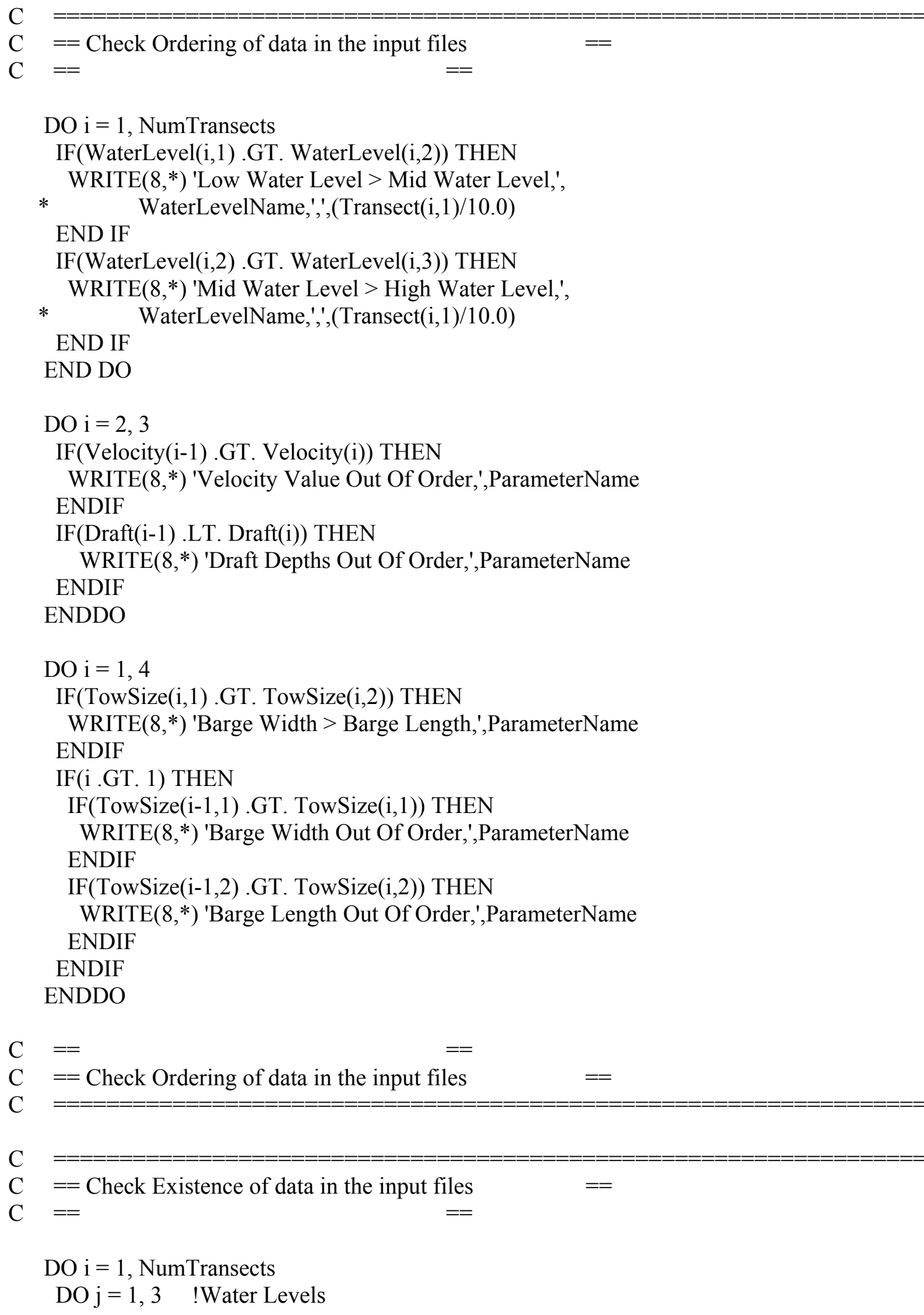




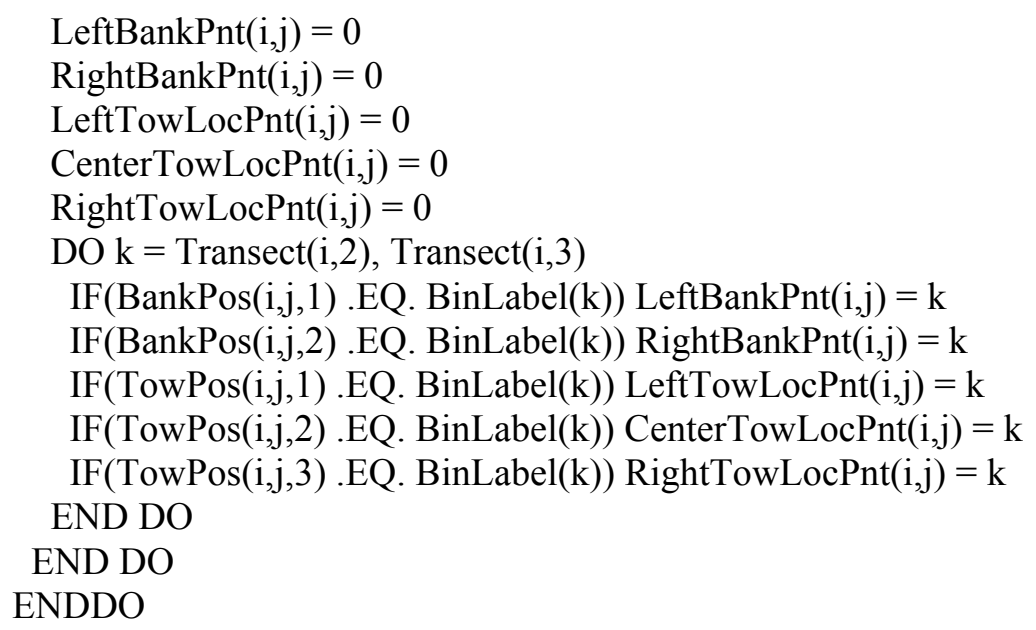


$\mathrm{C}$

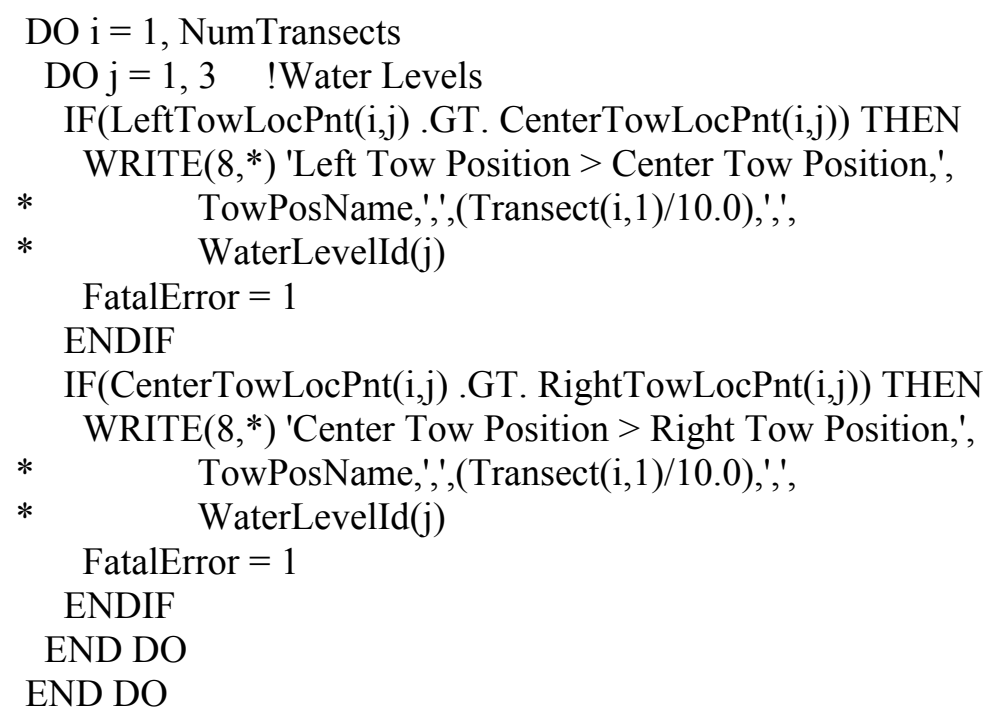


$\mathrm{C}$

IF (FatalError .EQ. 1) GOTO 999

$\mathrm{C}$

BoatHalfWidth $=\operatorname{INT}(($ TowSize $(4,1)+19.99) / 10.0) / 2$

c $* * * * *$ Calculate the width of each impact zone $* * * * *$

DO $\mathrm{i}=1$, NumTransects

Distance $($ Transect $(\mathrm{i}, 2))=0.0$

Width $($ Transect $(\mathrm{i}, 2))=0.0$

DO j $=($ Transect $(i, 2)+1)$, Transect $(i, 3)$

Distance $(\mathrm{j})=$ ABS $($ SQRT (

* $\quad(($ Easting(Transect(i,2))-Easting(j) $) * * 2.0)+$

* $\quad(($ Northing(Transect(i,2))-Northing(j))**2.0)))

$\operatorname{Width}(\mathrm{j})=\operatorname{Distance}(\mathrm{j})-\operatorname{Distance}(\mathrm{j}-1)$

ENDDO

ENDDO

DO $i=1$, NumTransects

DO $\mathrm{j}=$ LeftBankPnt $(\mathrm{i}, 3)+1$, RightBankPnt $(\mathrm{i}, 3)$

IF(Width(j) .GT. 17.5) THEN

WRITE $\left(8,{ }^{*}\right)$ 'Impact Zone Separation > 17.5 M,',

* CrossSectionName,',',BinLabel(j)

ENDIF

IF(Width(j) .LT. 5.0) THEN

WRITE $\left(8,{ }^{*}\right)$ 'Impact Zone Separation < 5.0 M,',

* CrossSectionName, ',',BinLabel(j)

ENDIF

END DO

END DO

$\mathrm{C}$

DO $i=1$, NumTransects

DO $\mathrm{j}=1,3 \quad$ !Water Levels

DO k = LeftBankPnt(i,j), RightBankPnt( $i, j)$

WaterDepth $(\mathrm{j}, \mathrm{k})=$ WaterLevel $(\mathrm{i}, \mathrm{j})-\operatorname{MSL}(\mathrm{k})$

END DO

END DO

END DO

DO $\mathrm{i}=1$, NumTransects

DO $\mathrm{j}=1,3 \quad$ !Water Levels

DepthError $=0$

TabsError $=0$

DO k = LeftBankPnt(i,j), RightBankPnt(i,j)

IF(WaterDepth(j,k) .LE. 0.0) THEN

WRITE $(8, *)$ 'Negative Water Depth,', 


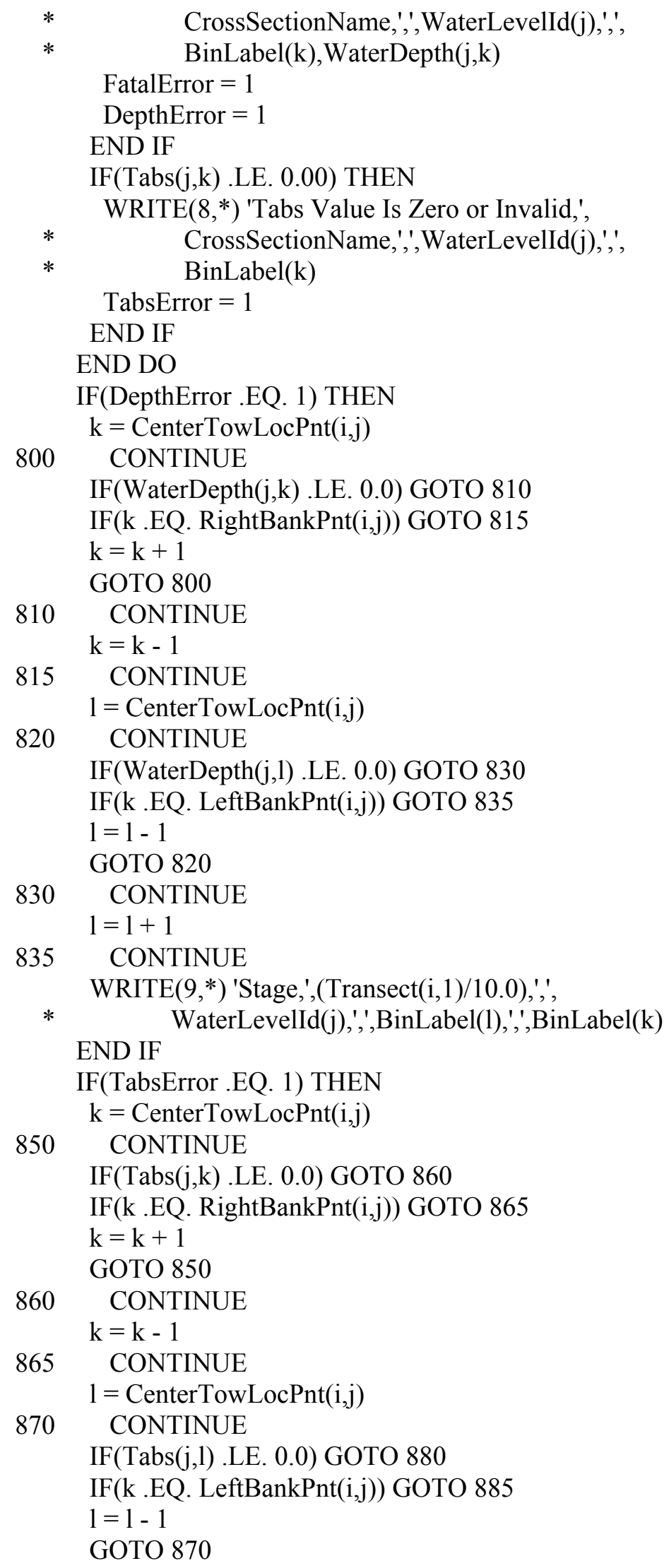




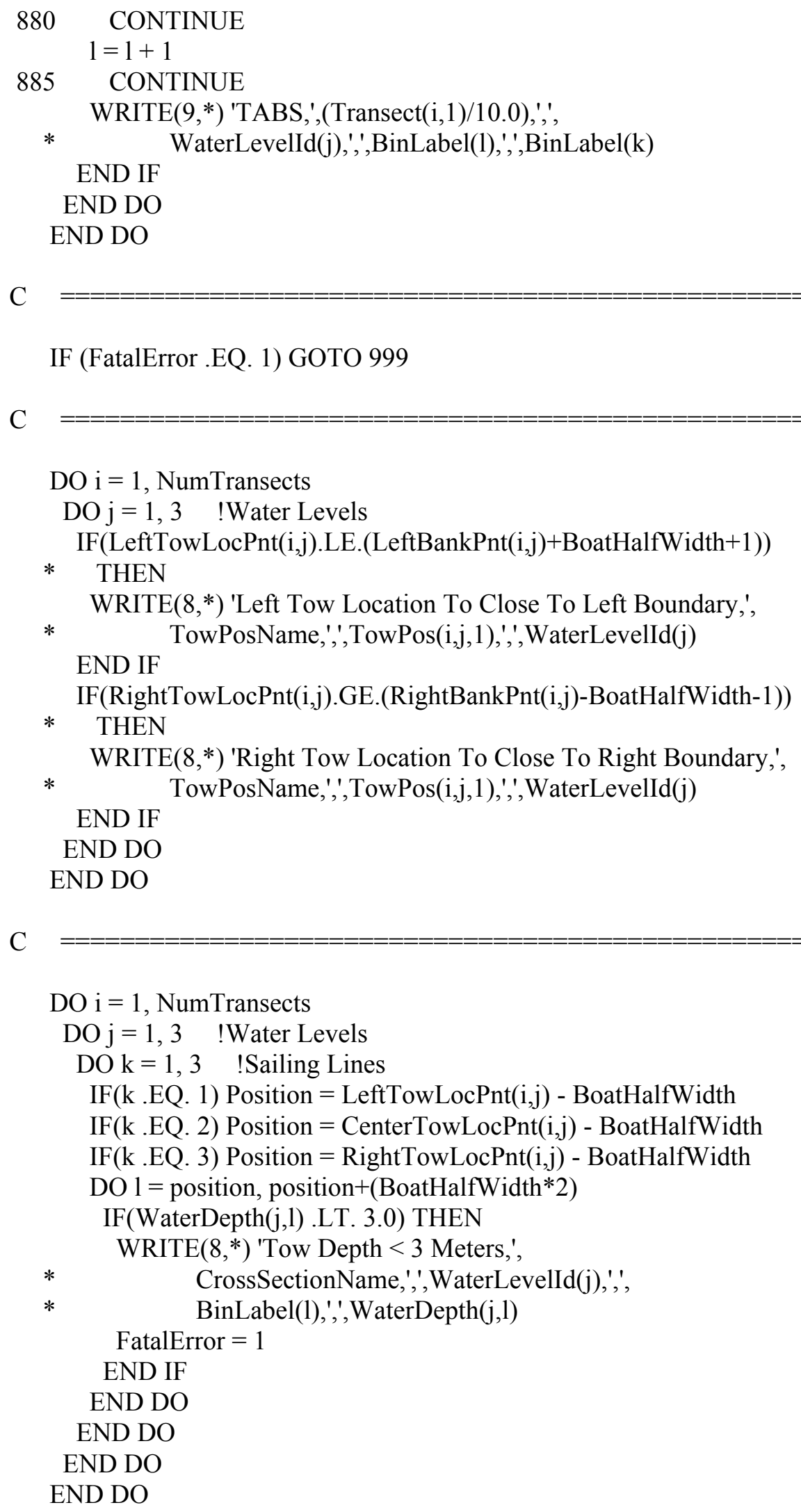


C

999 CONTINUE

CLOSE(1)

CLOSE(8)

CLOSE(9)

\begin{tabular}{|c|c|c|}
\hline$==$ & Program Termination Point & $==$ \\
\hline$==$ & $=$ & \\
\hline \multicolumn{3}{|c|}{$\begin{array}{l}\text { STOP ' NORMAL STOP CONDITIONS ' } \\
\text { END }\end{array}$} \\
\hline$==$ & $=$ & \\
\hline$==$ & Program Termination Point & $==$ \\
\hline
\end{tabular}




\title{
Appendix C NAVEFF Program
}

\section{Files}

\author{
Input \\ P\#_chan.dat \\ P\#_elev.dat \\ P\#_lmh.dat \\ P\#_parm.dat \\ P\#_sail.dat \\ P\# yz.trf \\ where: $\#=$ Pool Number \\ $\mathrm{y}=$ Stage, $\mathrm{z}=$ Sailing Line \\ Output \\ P\#_yz.out \\ where: \# = Pool Number \\ $\mathrm{y}=$ Stage, $\mathrm{z}=$ Sailing Line
}

\begin{tabular}{|l|l|l|l|l|}
\hline NAVEFF Output Variables & Type & Format & Example \\
\hline Variable & Description & Numeric & 5.1 & 523.5 \\
\hline RIVER_MI & River Mile & Character & 1 & U \\
\hline DIR & Upbound/Downbound & Character & 1 & F \\
\hline SPEED & Fast/Medium/Slow & Character & 1 & B \\
\hline SIZE & Big/Medium/Small & Character & 1 & L \\
\hline DRAFT & Load/Mixed/Empty & Character & 1 & O \\
\hline TURBINE & Open Wheel/Kort Nozzle & Character & 1 & H \\
\hline STAGE & High/Medium/Low & Character & 1 & L \\
\hline SL_POS & Left/Middle/Right & Character & 5 & UFBLO \\
\hline TRAFFIC & Variables 2 Thru 6 Concatenated & Character & 2 & HL \\
\hline STG_SL & Variables 7 and 8 Concatenated & Character & 10 & $305 \mathrm{~L} 5235$ \\
\hline CELL_ID & Cell ID & Numeric & 6.2 & 3.55 \\
\hline DEPTH & Depth (M) & Numeric & 7.3 & 0.205 \\
\hline VEL_CHANGE & Maximum Velocity Change (M/S) & Numeric & 7.3 & 0.107 \\
\hline DRAWDOWN & Drawdown (M) & Numeric & 7.1 & 20.8 \\
\hline SL_DIST & Distance from Sailing Line (M) & & \\
\hline
\end{tabular}




\begin{tabular}{|l|l|l|l|l|}
\hline NAVEFF Output Variables \\
\hline Variable & Description & Type & Format & Example \\
\hline SEC_WH & Secondary Wave Height (M) & Numeric & 7.3 & 0.107 \\
\hline MX_SCOUR & Maximum Scour (M) & Numeric & 9.4 & -0.1816 \\
\hline MX_SHEAR & Maximum Shear Stress (PA) & Numeric & 9.4 & 176.2432 \\
\hline AMB_FLUX & $\begin{array}{l}\text { Ambient Flux (Particle/Particle By } \\
\text { Vol.) }\end{array}$ & Numeric & 12.7 & 0.0001112 \\
\hline MX_AFLUX & $\begin{array}{l}\text { Maximum Ambient Flux } \\
\text { (Particle/Particle By Vol.) }\end{array}$ & Numeric & 12.7 & 0.2848123 \\
\hline AMB_SHEAR & Ambient Shear Stress (PA) & Numeric & 9.4 & 176.2432 \\
\hline
\end{tabular}

\section{Source Code}

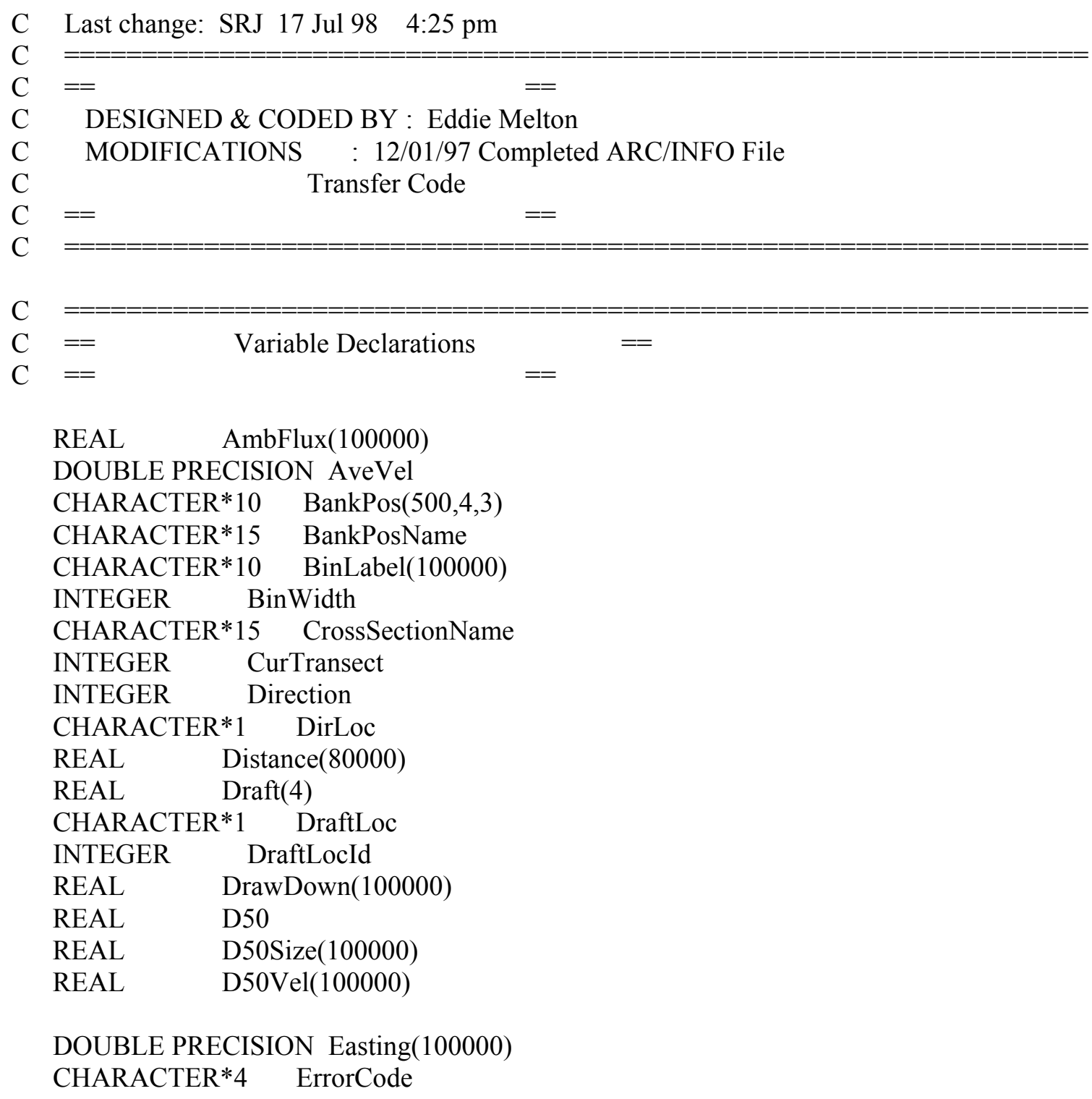

DOUBLE PRECISION Easting(100000)

CHARACTER *4 ErrorCode 


\begin{tabular}{|c|c|}
\hline INTEGER & ErrorCount \\
\hline REAL & astRiverMile \\
\hline DOUBLE PRECIS & ISION LeftArea \\
\hline INTEGER & LeftBankPnt \\
\hline INTEGER & LeftNear \\
\hline DOUBLE PRECIS & ISION LeftWidth \\
\hline REAL & ocDistance \\
\hline DOUBLE PRECIS & ISION MaxDepth \\
\hline REAL & $\operatorname{IaxFlux}(100000)$ \\
\hline INTEGER & MaxNumTransects \\
\hline INTEGER & MaxNumBins \\
\hline INTEGER & MaxRecords \\
\hline DOUBLE PRECIS & ISION MaxScour(100000) \\
\hline DOUBLE PRECIS & ISION NBNum \\
\hline DOUBLE PRECIS & ISION NBIncVal \\
\hline DOUBLE PRECIS & ISION MSL(100000) \\
\hline DOUBLE PRECIS & ISION Northing $(100000)$ \\
\hline INTEGER & NumTransects \\
\hline CHARACTER $* 15$ & 5 ParameterName \\
\hline CHARACTER $* 1$ & PoolLevel \\
\hline CHARACTER $* 3$ & PoolName \\
\hline REAL & elChange(100000) \\
\hline DOUBLE PRECIS & ISION RightArea \\
\hline INTEGER & RightBankPnt \\
\hline INTEGER & RightNear \\
\hline DOUBLE PRECIS & ISION RightWidth \\
\hline REAL & iverMile \\
\hline REAL & hearStress $(100000)$ \\
\hline REAL & hearAmb $(100000)$ \\
\hline CHARACTER $* 1$ & SizeLoc \\
\hline INTEGER & SizeLocID \\
\hline DOUBLE PRECIS & ISION Tabs $(4,100000)$ \\
\hline CHARACTER $* 1$ & TowLoc \\
\hline INTEGER & TowLocId \\
\hline INTEGER & TowLocPnt \\
\hline CHARACTER $* 10$ & $0 \quad$ TowPos $(500,4,4)$ \\
\hline CHARACTER $* 15$ & TowPosName \\
\hline REAL Tor & wSize $(5,3)$ \\
\hline CHARACTER $* 6$ & TrafficFile \\
\hline INTEGER & Transect $(500,4)$ \\
\hline REAL & elocity(4) \\
\hline CHARACTER $* 1$ & VelLoc \\
\hline INTEGER & VelLocId \\
\hline DOUBLE PRECIS & ISION WaterDepth(100000) \\
\hline REAL & aterLevel $(500,4)$ \\
\hline CHARACTER $* 15$ & 5 WaterLevelName \\
\hline INTEGER & CohSed \\
\hline INTEGER & CohClass \\
\hline
\end{tabular}


DOUBLE PRECISION ALEFT

DOUBLE PRECISION ALF

DOUBLE PRECISION ALFL

DOUBLE PRECISION ALFR

DOUBLE PRECISION AM

DOUBLE PRECISION ARIGHT

DOUBLE PRECISION ATOTAL

DOUBLE PRECISION B

DOUBLE PRECISION BE

DOUBLE PRECISION BLB

DOUBLE PRECISION BLEFT

DOUBLE PRECISION BRB

DOUBLE PRECISION BRIGHT

DOUBLE PRECISION BSIDE

DOUBLE PRECISION BTOTAL

DOUBLE PRECISION C

DOUBLE PRECISION CF

DOUBLE PRECISION D

DOUBLE PRECISION DE

DOUBLE PRECISION DEPTOW

DOUBLE PRECISION DISP

DOUBLE PRECISION FPV

DOUBLE PRECISION FV

DOUBLE PRECISION GRAV

DOUBLE PRECISION H

DOUBLE PRECISION L

DOUBLE PRECISION N

DOUBLE PRECISION NSIDEL

DOUBLE PRECISION NSIDER

DOUBLE PRECISION RL

DOUBLE PRECISION RTEM

DOUBLE PRECISION SCHIJF

DOUBLE PRECISION U1

DOUBLE PRECISION V

DOUBLE PRECISION VAM

DOUBLE PRECISION VDISP

DOUBLE PRECISION VFACTL

DOUBLE PRECISION VFACTR

REAL $\quad \mathrm{VG}$

DOUBLE PRECISION VL

DOUBLE PRECISION VLIMRAT

DOUBLE PRECISION VLN

DOUBLE PRECISION VLO

REAL VNU

DOUBLE PRECISION VW

DOUBLE PRECISION VRAL

DOUBLE PRECISION VRAR

DOUBLE PRECISION VRLM

DOUBLE PRECISION VRM

DOUBLE PRECISION VRRM 
real $Y$

DOUBLE PRECISION yy

DOUBLE PRECISION $Z$

DOUBLE PRECISION ZALF

DOUBLE PRECISION ZALFL

DOUBLE PRECISION ZALFR

DOUBLE PRECISION ZC

DOUBLE PRECISION ZSL

DOUBLE PRECISION ZSM

DOUBLE PRECISION ZSML

DOUBLE PRECISION ZSMR

DOUBLE PRECISION ZSR

DOUBLE PRECISION ZT

CHARACTER $* 1 \quad$ KOLoc

INTEGER KOLocId

DOUBLE PRECISION A

DOUBLE PRECISION BB

DOUBLE PRECISION CDECAY

DOUBLE PRECISION CEXP

DOUBLE PRECISION CFFACTOR

DOUBLE PRECISION CFLOW

DOUBLE PRECISION CFUNC

DOUBLE PRECISION cj

DOUBLE PRECISION CJTEMP

DOUBLE PRECISION coef

DOUBLE PRECISION $\mathrm{cp}$

DOUBLE PRECISION CPARA

DOUBLE PRECISION CPZ1

DOUBLE PRECISION CPZ2

DOUBLE PRECISION D0

DOUBLE PRECISION delcf

REAL DEP

DOUBLE PRECISION DEPTMP

REAL DP

DOUBLE PRECISION E

DOUBLE PRECISION FPX

DOUBLE PRECISION FRES

DOUBLE PRECISION FUNC

DOUBLE PRECISION FX

DOUBLE PRECISION HP

DOUBLE PRECISION HPN

DOUBLE PRECISION HPO

DOUBLE PRECISION K11

DOUBLE PRECISION KKK

DOUBLE PRECISION LBARGES

DOUBLE PRECISION nu

INTEGER NUMX

INTEGER NUMY 
DOUBLE PRECISION P1DECAY

DOUBLE PRECISION P2DECAY

REAL PSPACE

DOUBLE PRECISION PUSH

DOUBLE PRECISION PUSH1

DOUBLE PRECISION PUSH2

DOUBLE PRECISION PUSH3

DOUBLE PRECISION RHO

DOUBLE PRECISION RR

DOUBLE PRECISION $\mathrm{S}$

DOUBLE PRECISION SETBACK

REAL TBL

DOUBLE PRECISION temp1

REAL THRUST

DOUBLE PRECISION THRUSTP

DOUBLE PRECISION TIHP

DOUBLE PRECISION U2

DOUBLE PRECISION V0

DOUBLE PRECISION V1

DOUBLE PRECISION V2

REAL VA

DOUBLE PRECISION VBDMAX

DOUBLE PRECISION VDIRECT

DOUBLE PRECISION VELLOCC

DOUBLE PRECISION VLM(5)

DOUBLE PRECISION VMAXTEST

DOUBLE PRECISION VMPH

DOUBLE PRECISION VR

DOUBLE PRECISION VRES

DOUBLE PRECISION VTEST

DOUBLE PRECISION vtotal

DOUBLE PRECISION VWAKEgX

DOUBLE PRECISION vwakamax

DOUBLE PRECISION VXMAX

DOUBLE PRECISION VXRL

DOUBLE PRECISION VXRPROP

DOUBLE PRECISION VXRR

DOUBLE PRECISION $X$

DOUBLE PRECISION XBEGIN

DOUBLE PRECISION XCALC

DOUBLE PRECISION XPROP

DOUBLE PRECISION XSPACE

DOUBLE PRECISION YL

DOUBLE PRECISION YR

DOUBLE PRECISION YSPACE

DOUBLE PRECISION ZZB

DOUBLE PRECISION AB

DOUBLE PRECISION alpha

DOUBLE PRECISION SecWaveHgt(100000) 
DOUBLE PRECISION HSave1

DOUBLE PRECISION HSave2

CHARACTER AKO*5

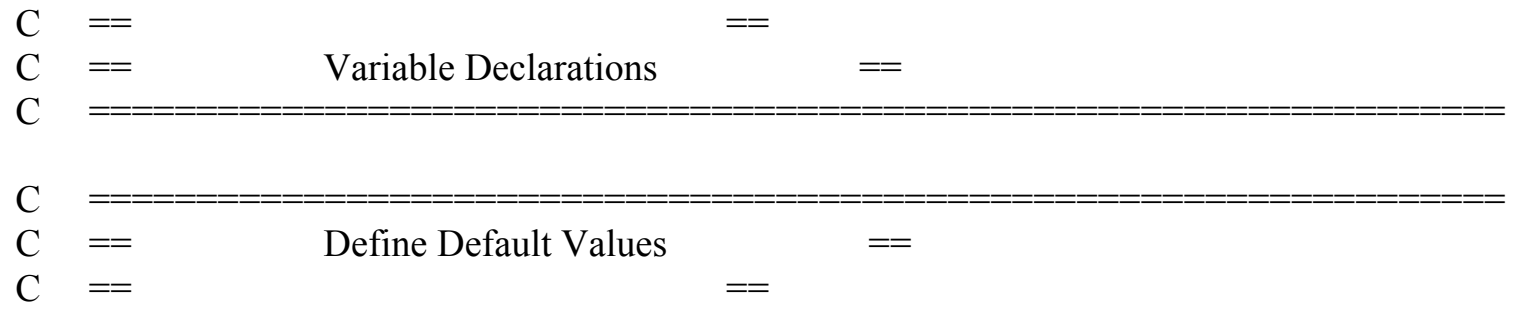

DATA MaxNumTransects / 100 /

DATA MaxNumBins / 800 /

MaxRecords $=$ MaxNumTransects $*$ MaxNumBins

NumTransects $=$ MaxNumTransects

ErrorCount $=0$

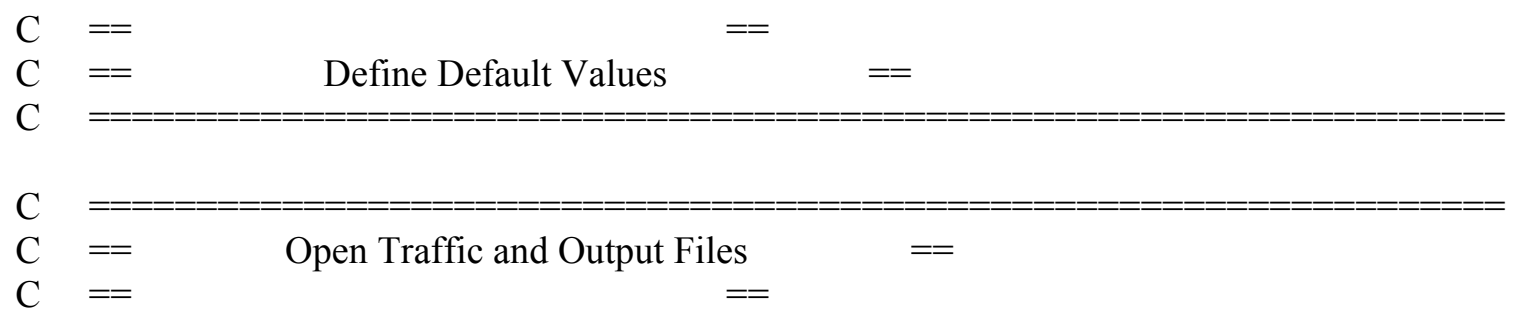

WRITE $(*, *)$ 'Enter Traffic File Name: '

$\operatorname{READ}(*, *)$ TrafficFile

PoolName $=$ TrafficFile $(1: 3)$

OPEN $(1$, FILE $=$ TrafficFile//'.trf', STATUS='old')

$\operatorname{OPEN}(8$, FILE $=$ TrafficFile//'.err', STATUS='new')

OPEN(9, FILE=TrafficFile//'.out', STATUS='new')

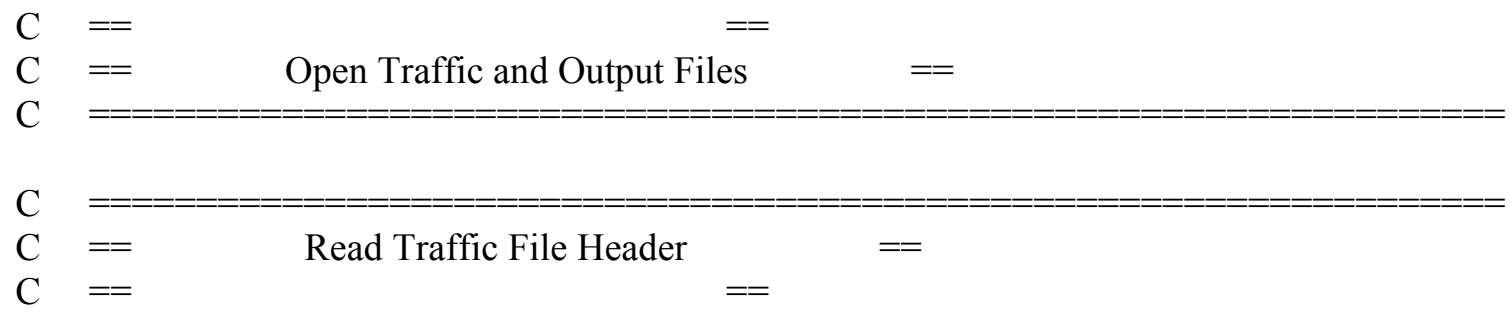

WRITE $\left(8,{ }^{*}\right)$ 'ErrCode,Trans,Dir,Speed,Size,Draft,K/O,Stage,Sail,Traf

*fic,Stg_Sl,TotArea,_LftArea,TotWth,LftWth,TowWth,TowLen,Tow Draft,V

*,Vamb,MaxDepth,HBefore,Hafter,RetVel,DrwDwn' 


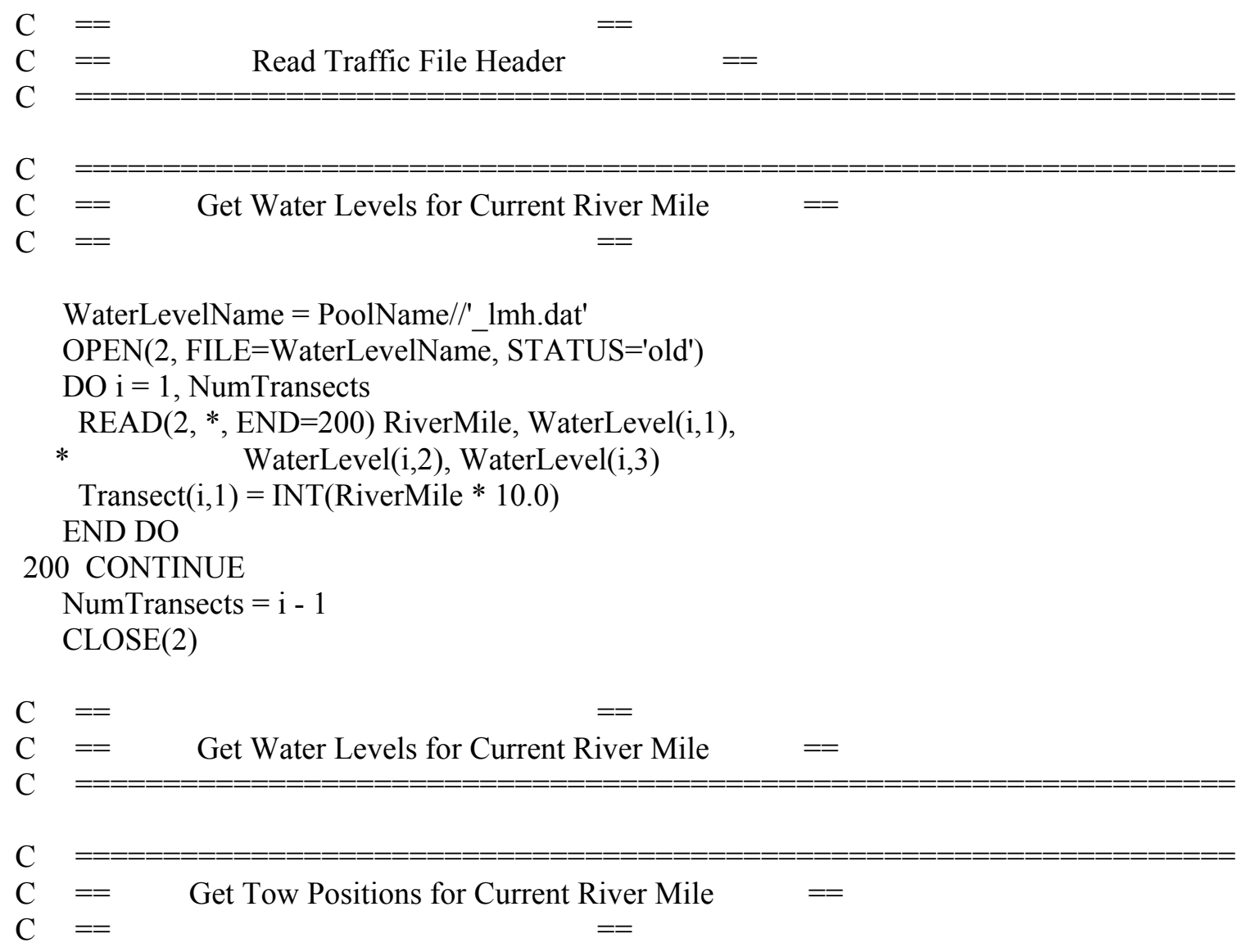

TowPosName $=$ PoolName//'_sail.dat' OPEN(3, FILE=TowPosName, STATUS='old')

DO $\mathrm{i}=1$, NumTransects $\operatorname{READ}(3,350, \mathrm{END}=300)$ RiverMile, * TowPos(i,1,1),TowPos(i,1,2),TowPos(i,1,3), * TowPos(i,2,1),TowPos(i,2,2),TowPos(i,2,3),

* TowPos(i,3,1),TowPos(i,3,2),TowPos(i,3,3)

END DO

300 CONTINUE

CLOSE(3)

350 FORMAT(f5.1, a10, a10, a10, a10, a10, a10, a10, a10, a10)

$\mathrm{C}==$

$\mathrm{C}==\quad$ Get Tow Positions for Current River Mile $==$

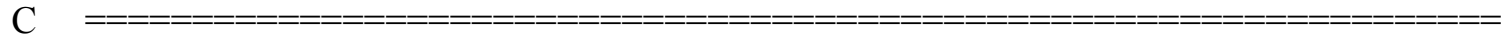

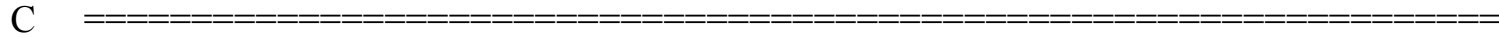

$\mathrm{C}==$ Get Bank Positions for Current River Mile $==$

$\mathrm{C}==\quad==$

BankPosName $=$ PoolName//'_chan.dat'

$\operatorname{OPEN}(4$, FILE $=$ BankPosName, STATUS='old')

DO $\mathrm{i}=1$, NumTransects 


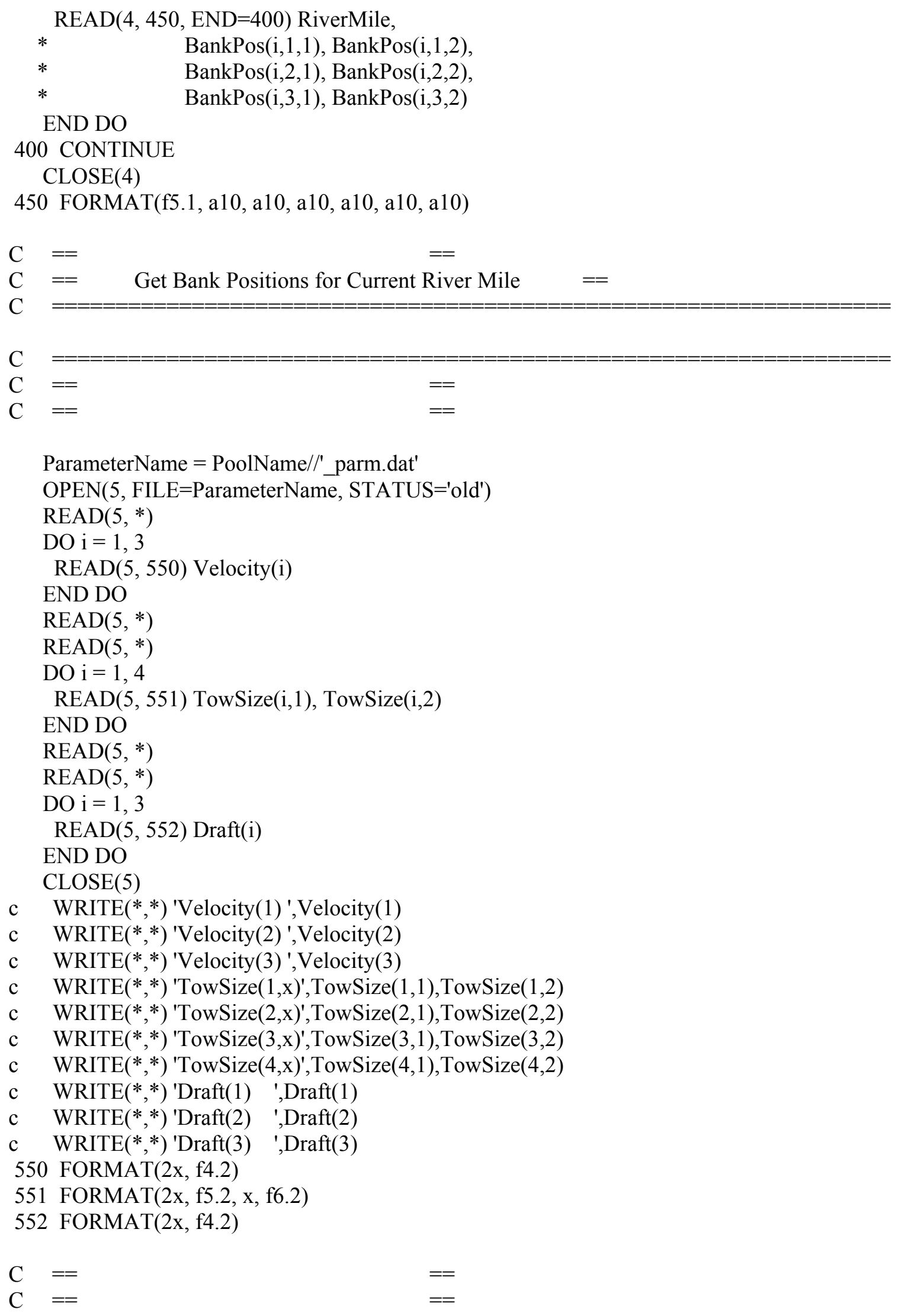




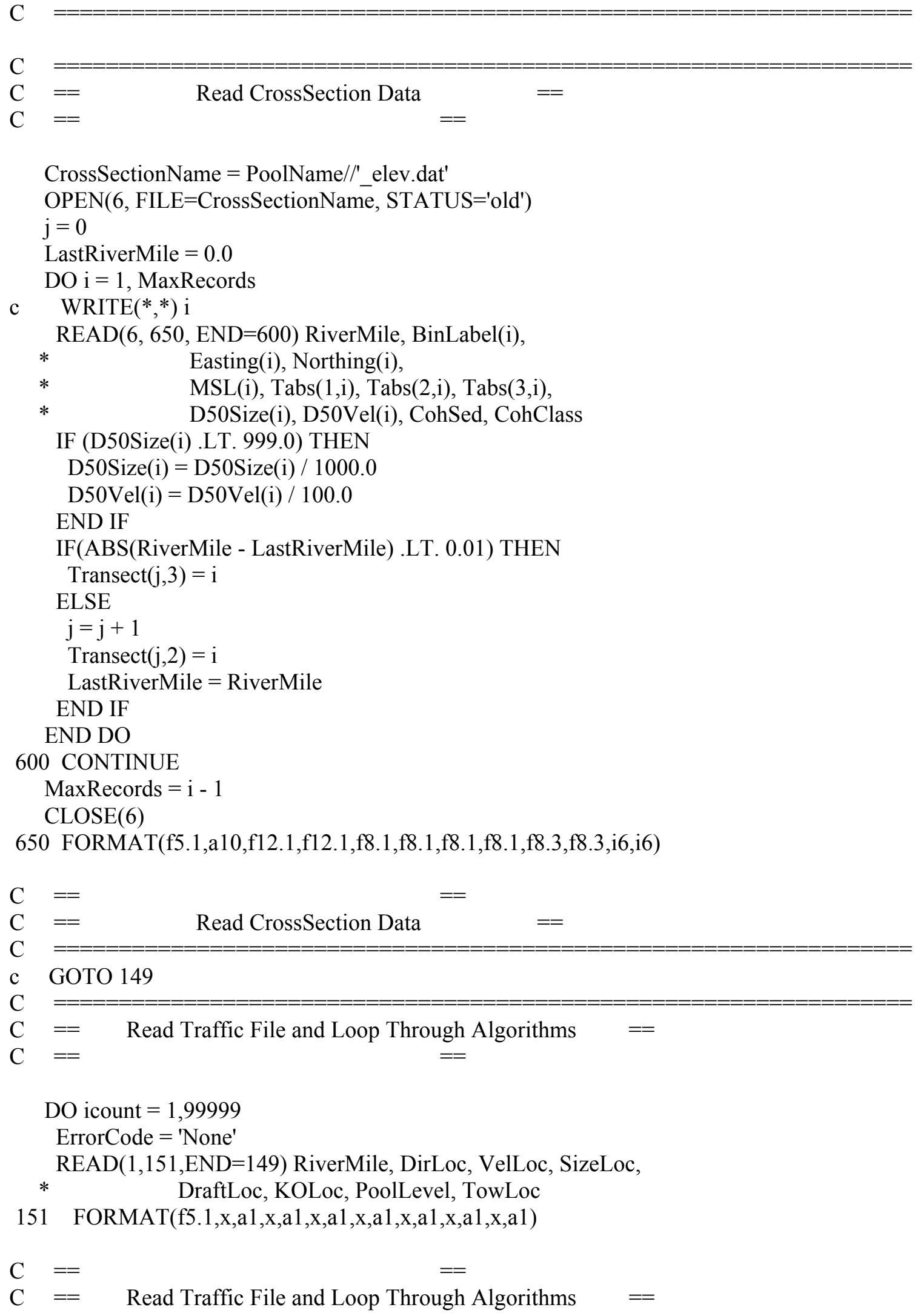




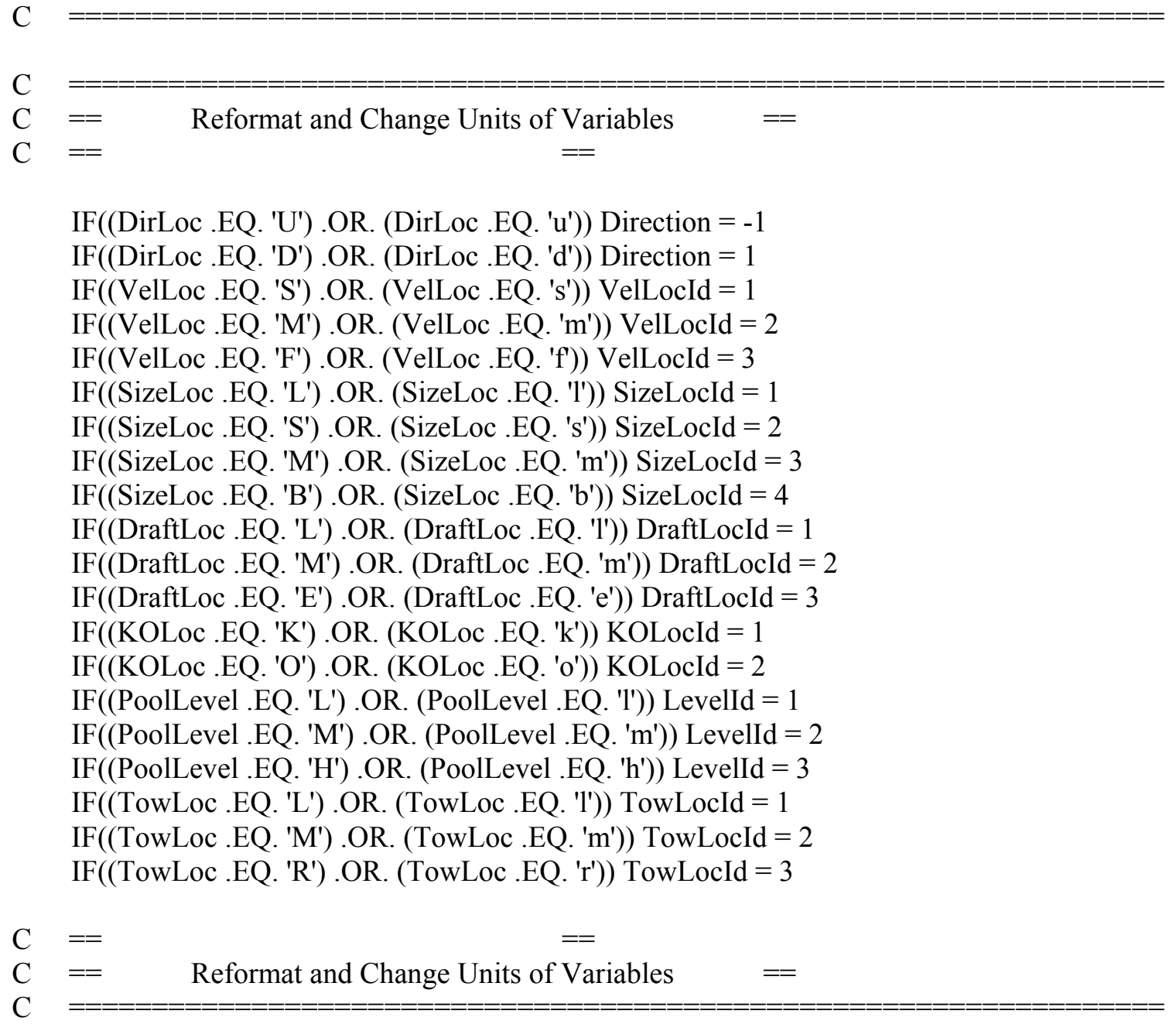

DO $\mathrm{i}=1$,NumTransects

IF(INT(RiverMile * 10.0) .EQ. Transect(i,1)) GOTO 100

END DO

GOTO 859

100 CONTINUE

CurTransect $=\mathrm{i}$

WRITE(*,*) 'Transect',RiverMile,'(',icount,')','[',ErrorCount,']'

TowLocPnt $=0$

LeftBankPnt $=0$

RightBankPnt $=0$

DO $\mathrm{i}=$ Transect(CurTransect,2), Transect(CurTransect,3)

WaterDepth(i) $=$ WaterLevel(CurTransect,LevelId) - MSL(i)

$\operatorname{IF}($ WaterDepth(i) .LT. 0.0) WaterDepth(i) $=0.0$

$\mathrm{c}$

*** Find Tow Location ***

IF(TowPos(CurTransect,LevelId,TowLocId) .EQ. BinLabel(i))

* TowLocPnt $=\mathrm{i}$

IF(BankPos(CurTransect,LevelId,1) .EQ. BinLabel(i))

* $\quad$ LeftBankPnt $=\mathrm{i}$ 
IF(BankPos(CurTransect,LevelId,2) .EQ. BinLabel(i))

* $\quad$ RightBankPnt = i

END DO

$\begin{array}{ll}\text { c } & \text { DO i }=\text { LeftBankPnt, RightBankPnt } \\ \text { c } & \left.\text { IF(WaterDepth(i).EQ. 0.0) WRITE(8, }{ }^{*}\right) \text { BinLabel(i),,','LevelId } \\ \text { c } & \text { END DO }\end{array}$

IF(TowLocPnt .EQ. 0) GOTO 860

IF(LeftBankPnt .EQ. 0) GOTO 861

IF(RightBankPnt .EQ. 0) GOTO 862

c

*** Calculate Areas ***

LeftArea $=0.0$

RightArea $=0.0$

DO $\mathrm{i}=$ TowLocPnt, LeftBankPnt, -1

LeftArea $=$ LeftArea $+($ WaterDepth $(i) * 10.0)$

END DO

DO $\mathrm{i}=$ TowLocPnt, RightBankPnt

RightArea $=$ RightArea $+($ WaterDepth(i) $* 10.0)$

END DO

c $\quad * * *$ Adjust Areas for the Tow Location ***

LeftArea $=$ LeftArea $-($ WaterDepth $($ TowLocPnt $) * 10.0 / 2.0)$

RightArea $=$ RightArea $-($ WaterDepth $($ TowLocPnt $) * 10.0 / 2.0)$

AveVel $=0.0$

MaxDepth $=0.0$

DO i = LeftBankPnt, RightBankPnt

AveVel $=$ AveVel $+($ Tabs $($ LevelId,i $) *($ WaterDepth $(i) * 10.0))$

IF(WaterDepth(i) .GT. MaxDepth) MaxDepth $=$ WaterDepth(i)

Distance $(\mathrm{i})=\mathrm{ABS}(\mathrm{SQRT}((($ Easting $($ TowLocPnt $)$-Easting(i) $) * * 2.0)$

* + ((Northing(TowLocPnt)-Northing(i))**2.0)))

END DO

AveVel $=$ AveVel $/($ LeftArea + RightArea $)$

LeftWidth $=$ SQRT (

* $\quad(($ Easting(TowLocPnt)-Easting(LeftBankPnt) $) * * 2.0)+$

* ((Northing(TowLocPnt)-Northing(LeftBankPnt)) ** 2.0))

RightWidth $=$ SQRT (

* $\quad(($ Easting(TowLocPnt)-Easting(RightBankPnt) $) * * 2.0)+$

* $\quad$ ((Northing(TowLocPnt)-Northing(RightBankPnt) $) * * 2.0)$ )

BinWidth $=$ INT $($ TowSize $($ SizeLocId,1) $/$ 10.0)

LeftNear $=$ TowLocPnt - BinWidth

RightNear $=$ TowLocPnt + BinWidth

c $\quad$ GOTO 99

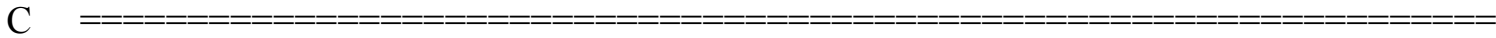

$\mathrm{C}==$ Start of SCHIJF Method from Visual Basic Code $==$ 
BTOTAL $=$ LeftWidth + RightWidth

BLEFT $=$ LeftWidth

ATOTAL $=$ LeftArea + RightArea

ALEFT $=$ LeftArea

$\mathrm{D}=\operatorname{Draft}($ DraftLocId $)$

B = TowSize(SizeLocId,1)

$\mathrm{L}=$ TowSize(SizeLocId,2)

DEPTOW $=$ WaterDepth(TowLocPnt)

IF(DEPTOW .LT. 3.0) GOTO 854

$\mathrm{VAM}=$ AveVel

$\mathrm{VW}=$ Velocity (VelLocId $)$

$\mathrm{VG}=\mathrm{VW}+\mathrm{VAM} *$ Direction

$\mathrm{V}=\mathrm{ABS}(\mathrm{VG}-($ Direction * $1.2 * \mathrm{VAM}))$

$\mathrm{GRAV}=9.805$

c

$* * *$ SET WATER VISCOSITY $=0.0000011 \mathrm{M}^{* * 2} / \mathrm{SEC}$ FOR TEMP $=17$ DEG C

$\mathrm{VNU}=0.0000011$

$\mathrm{c}$

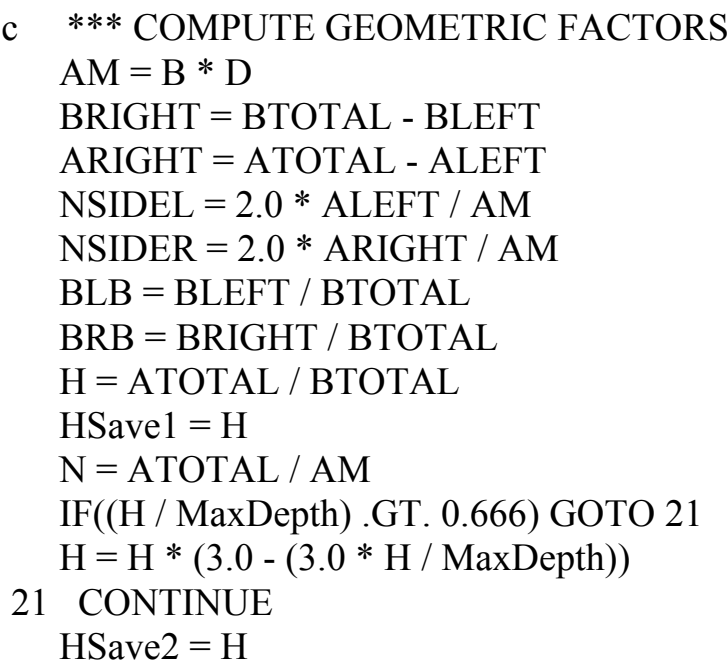

c SOLVE HOCHSTEIN EQUATION FOR U1 FOR DISPLACEMENT CALCULATION $\mathrm{U} 1=\mathrm{ABS}(\mathrm{V}) *((\mathrm{~N} /(\mathrm{N}-1)) * * 1.25-1)$

c $\quad * * *$ COMPUTE DISPLACEMENT THICKNESS

50 continue

$\mathrm{VDISP}=\mathrm{ABS}(\mathrm{V})+\mathrm{U} 1$

$\mathrm{RL}=\mathrm{VDISP} * \mathrm{~L} / \mathrm{VNU}$

$\mathrm{DISP}=0.292 * \mathrm{~L} /(0.43429 * \mathrm{LOG}(\mathrm{ABS}(\mathrm{RL}))) * * 2.58$

$\mathrm{DE}=\mathrm{D}+\mathrm{DISP}$

$\mathrm{BE}=\mathrm{B}+2.0 * \mathrm{DISP}$

$\mathrm{N}=$ ATOTAL $/ \mathrm{BE} / \mathrm{DE}$ 
c

LoopCnt $=1$

$\mathrm{VLO}=\mathrm{ABS}(\mathrm{V})$

54 continue

$\mathrm{RTEM}=\mathrm{VLO} * * 2.0 / \mathrm{GRAV} / \mathrm{H}$

$\mathrm{FV}=1.0-1.0 / \mathrm{N}+0.5 *$ RTEM $-1.5 *$ RTEM ** $(1.0 / 3.0)$

$\mathrm{FPV}=\mathrm{VLO} / \mathrm{GRAV} / \mathrm{H}-(\mathrm{VLO} * *(-1.0 / 3.0)) /$

* $\quad(\mathrm{GRAV} * \mathrm{H}) * *(1.0 / 3.0)$

$\mathrm{VLN}=\mathrm{VLO}-\mathrm{FV} / \mathrm{FPV}$

IF (ABS((VLO - VLN) / VLO) .LT. 0.0001) GOTO 55

LoopCnt $=$ LoopCnt +1

$\mathrm{VLO}=\mathrm{VLN}$

IF(LoopCnt .GE. 100) GOTO 853

GOTO 54

55 continue

$\mathrm{VL}=\mathrm{VLN}$

C THIS ROUTINE FINDS U1 FOR V $>.95 *$ VL

IF (V .LT. 0.95*VL) GOTO 57

$\mathrm{VTEMP}=.95 * \mathrm{VL}$

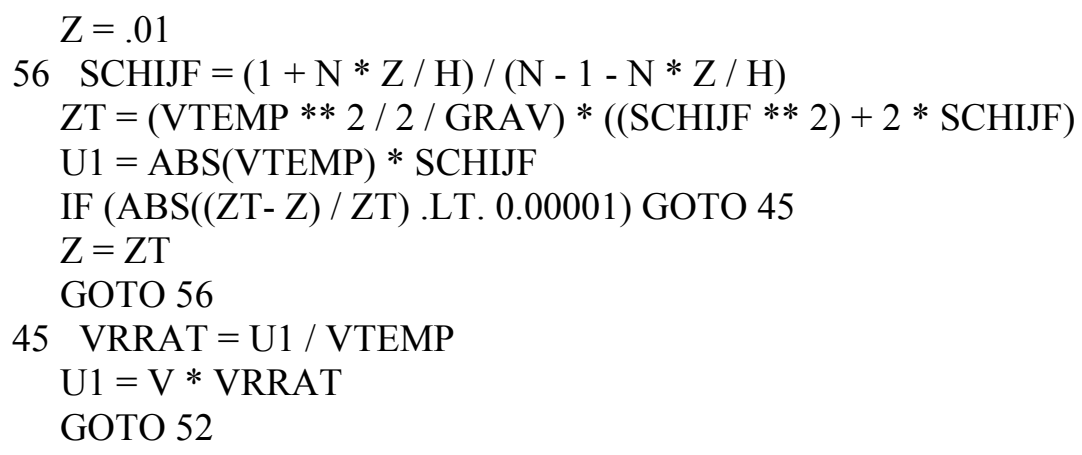

c $\quad * * *$ SOLVE SCHIJF EQUATION FOR RETURN VELOCITY

57 LoopCnt $=1$

$\mathrm{Z}=0.01$

$51 \mathrm{SCHIJF}=(1.0+\mathrm{N} * \mathrm{Z} / \mathrm{H}) /(\mathrm{N}-1.0-\mathrm{N} * \mathrm{Z} / \mathrm{H})$

$\mathrm{ZT}=(\mathrm{V} * * 2.0 / 2.0 / \mathrm{GRAV}) *((\mathrm{SCHIJF} * * 2.0)+2.0 * \mathrm{SCHIJF})$

$\mathrm{U} 1=\mathrm{ABS}(\mathrm{V}) * \mathrm{SCHIJF}$

IF (ABS((ZT - Z) / ZT) .LT. 0.00001) GOTO 52

LoopCnt $=$ LoopCnt +1

$\mathrm{Z}=\mathrm{ZT}$

IF(LoopCnt .GE. 100) GOTO 852

GOTO 51

52 CONTINUE

VLIMRAT $=\mathrm{ABS}(\mathrm{V}) / \mathrm{VL}$

c $\quad * * *$ APPLY CORRECTION FACTOR

61 continue 


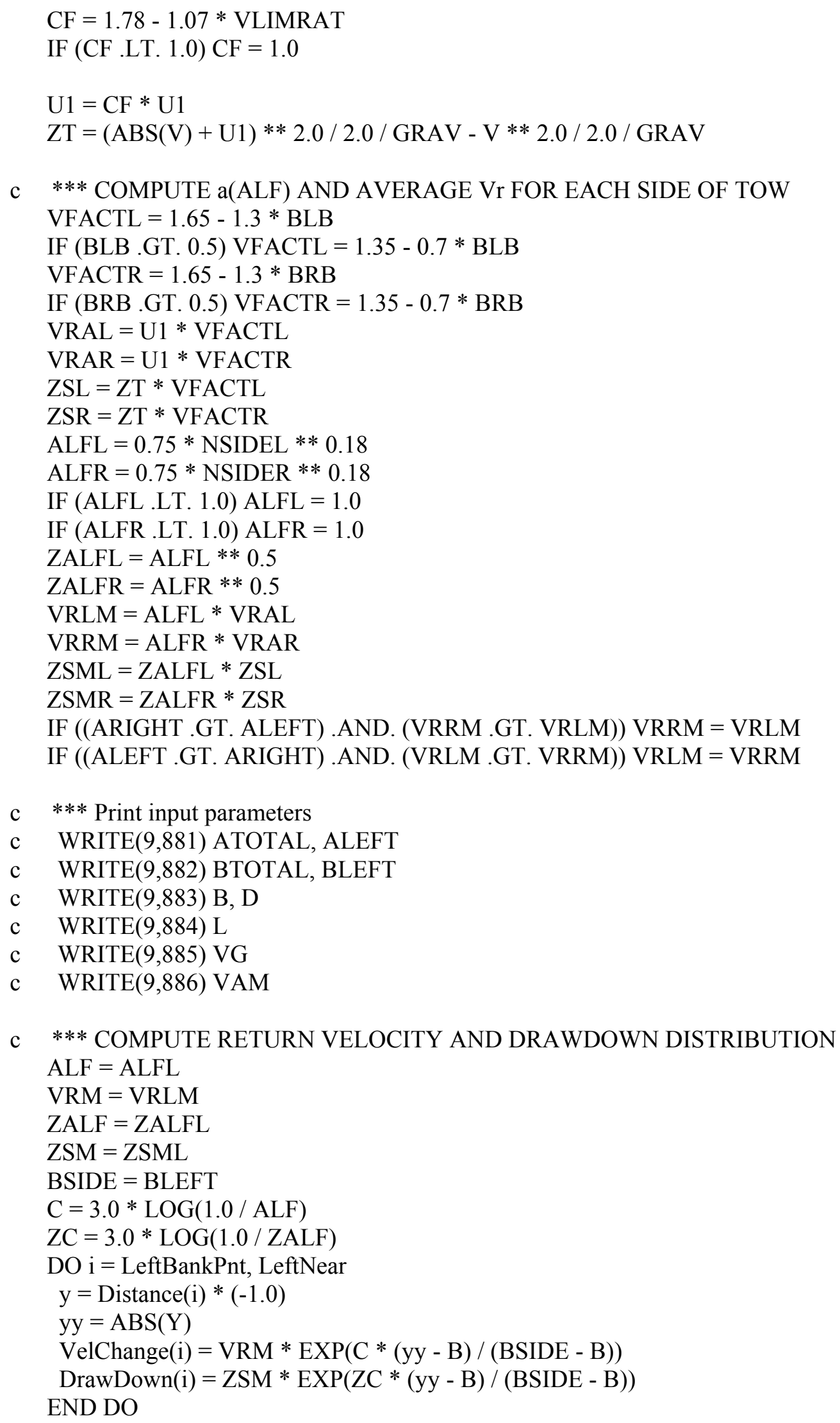




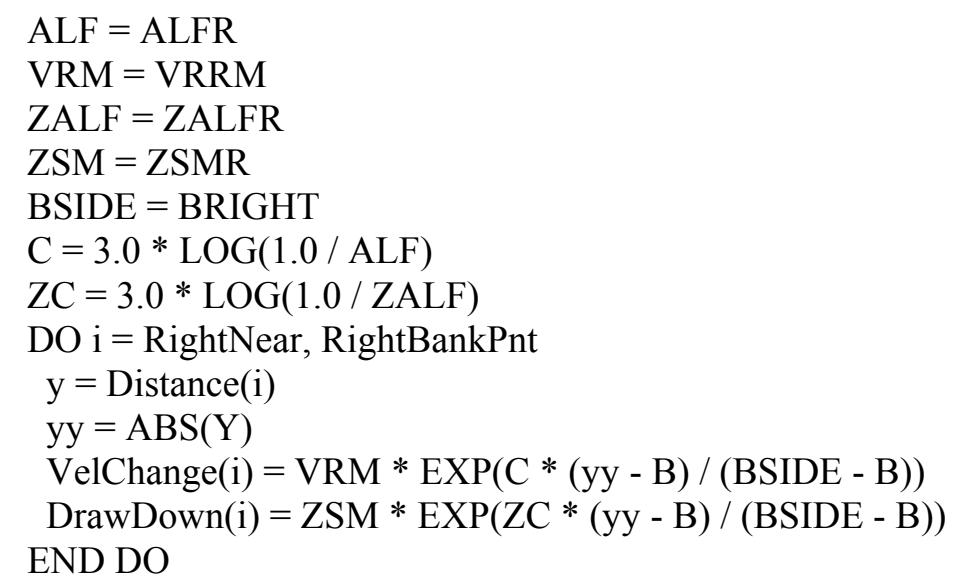




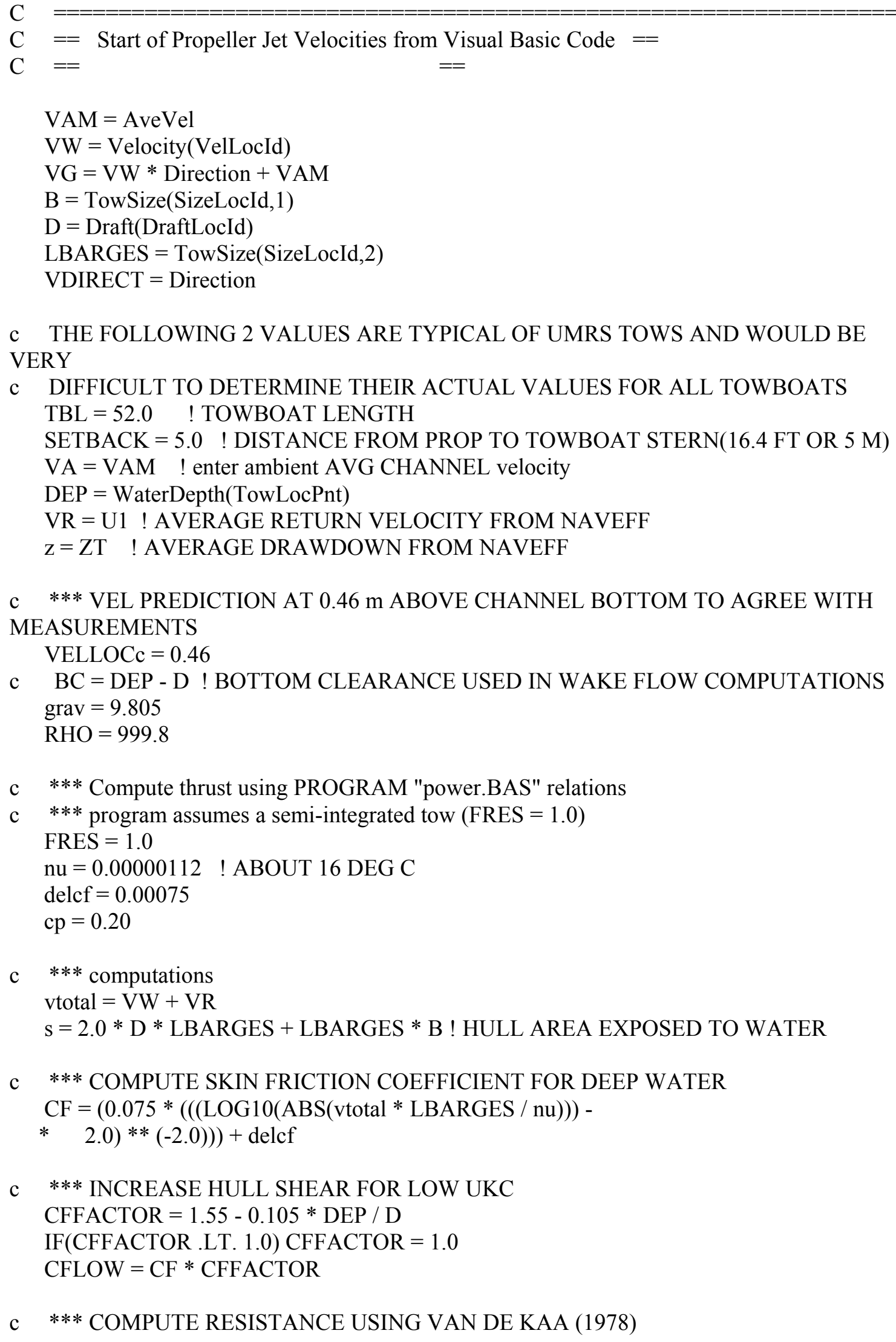




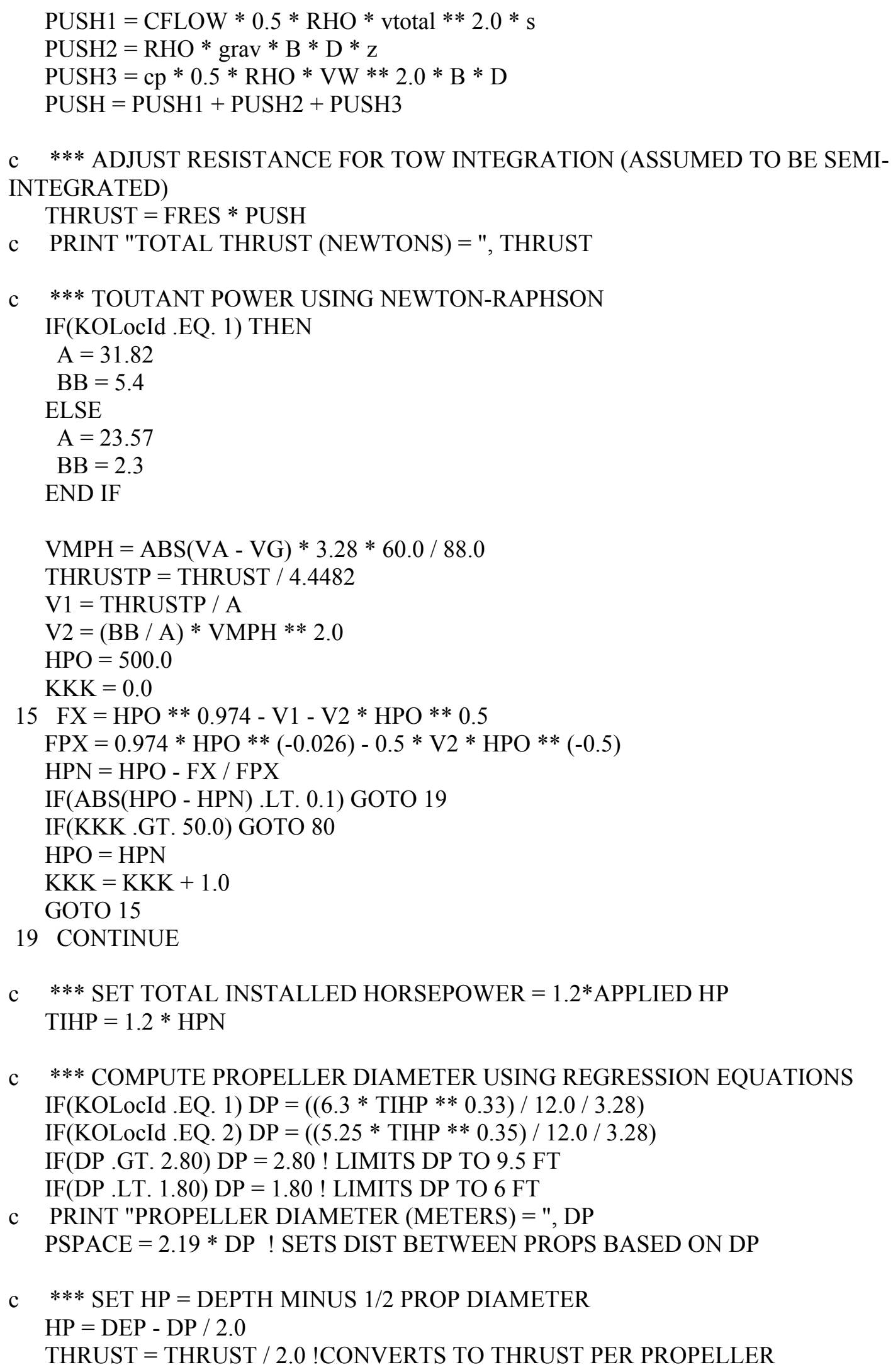


c $\quad * * *$ X IS MEASURED FROM BOW OF BARGES

XBEGIN = LBARGES + TBL - 20.0 !BEGIN LOOKING FOR MAX 20M AHEAD OF STERN

$\mathrm{XSPACE}=1.9$

NUMX $=200$

c $\quad * * *$ Y IS MEASURED LATERALLY FROM CENTER OF TOWBOAT

$\mathrm{Y}=3.0 \quad$ ! $\mathrm{Y}=0 \mathrm{M}$ IS REPRESENTED BY VEL AT $\mathrm{Y}=3 \mathrm{M}$ BECAUSE PEAK AT $3 \mathrm{M}$

c $* * *$ CAN BE SUBSTANTIALLY GREATER THAN AT Y=0 AT SHALLOW DEPTHS

YSPACE $=10.0$

$\mathrm{NUMY}=3$

c $\quad * * *$ MISCELLANEOUS INPUT

c $\quad * * *$ INPUT APPLICABLE TO BOTH PROPELLER TYPES

CDECAY $=0.34$

$\mathrm{P} 1 \mathrm{DECAY}=0.93$

$\mathrm{P} 2 \mathrm{DECAY}=0.24$

IF(KOLocId .EQ. 1) GOTO 70

c *** THIS SECTION FOR SETTING OPEN WHEEL PARAMETERS

$\mathrm{D} 0=0.71 * \mathrm{DP}$

$\mathrm{E}=0.43$

$\mathrm{CPARA}=0.12 *(\mathrm{DP} / \mathrm{HP}) * * 0.6666$

$\mathrm{CEXP}=0.656$

$\mathrm{CFUNC}=0.5$

GOTO 190

70 CONTINUE

c $\quad * * *$ THIS SECTION FOR SETTING KORT NOZZLE PARAMETERS

$\mathrm{D} 0=\mathrm{DP}$

$\mathrm{E}=0.58$

CPARA $=0.04$

$\mathrm{CEXP}=0.85$

$\mathrm{CFUNC}=0.25$

c $\quad * * *$ END OF KORT VERSUS OPEN

190 CONTINUE

c $\quad * * *$ end input

c $\quad * * *$ COMPUTE VELOCITY EXITING PROPELLER

$\mathrm{V} 0=1.13 / \mathrm{D} 0 *($ THRUST $/$ RHO $) * * 0.5$

$\mathrm{U} 2=\mathrm{V} 0$

IF(U2 .EQ. 0.0) U2 $=0.00001$

c PRINT "VELOCITY EXITING PROPELLER (METERS/SEC) = ", U2

c $\quad * * *$ BEGIN ITERATION LOOP FOR X AND Y

DO J $=1$, NUMY

IF(J .EQ. 2) Y $=10.0$ !THIS RESETS LOCATION TO 10 M INCREMENTS

$\mathrm{X}=\mathrm{XBEGIN} ! \mathrm{x}=0$ at bow of barges

$\mathrm{VTEST}=0.0$ 


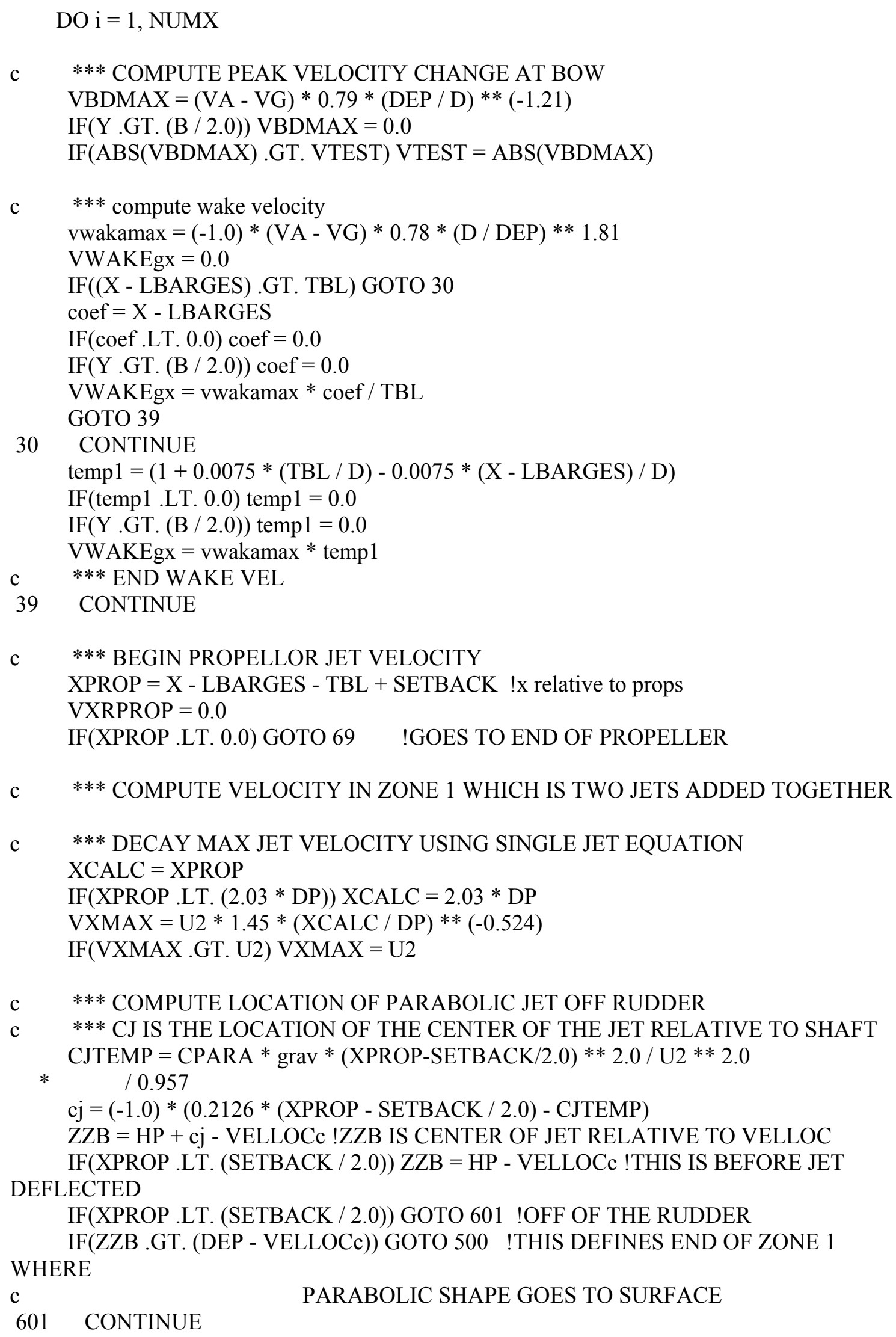




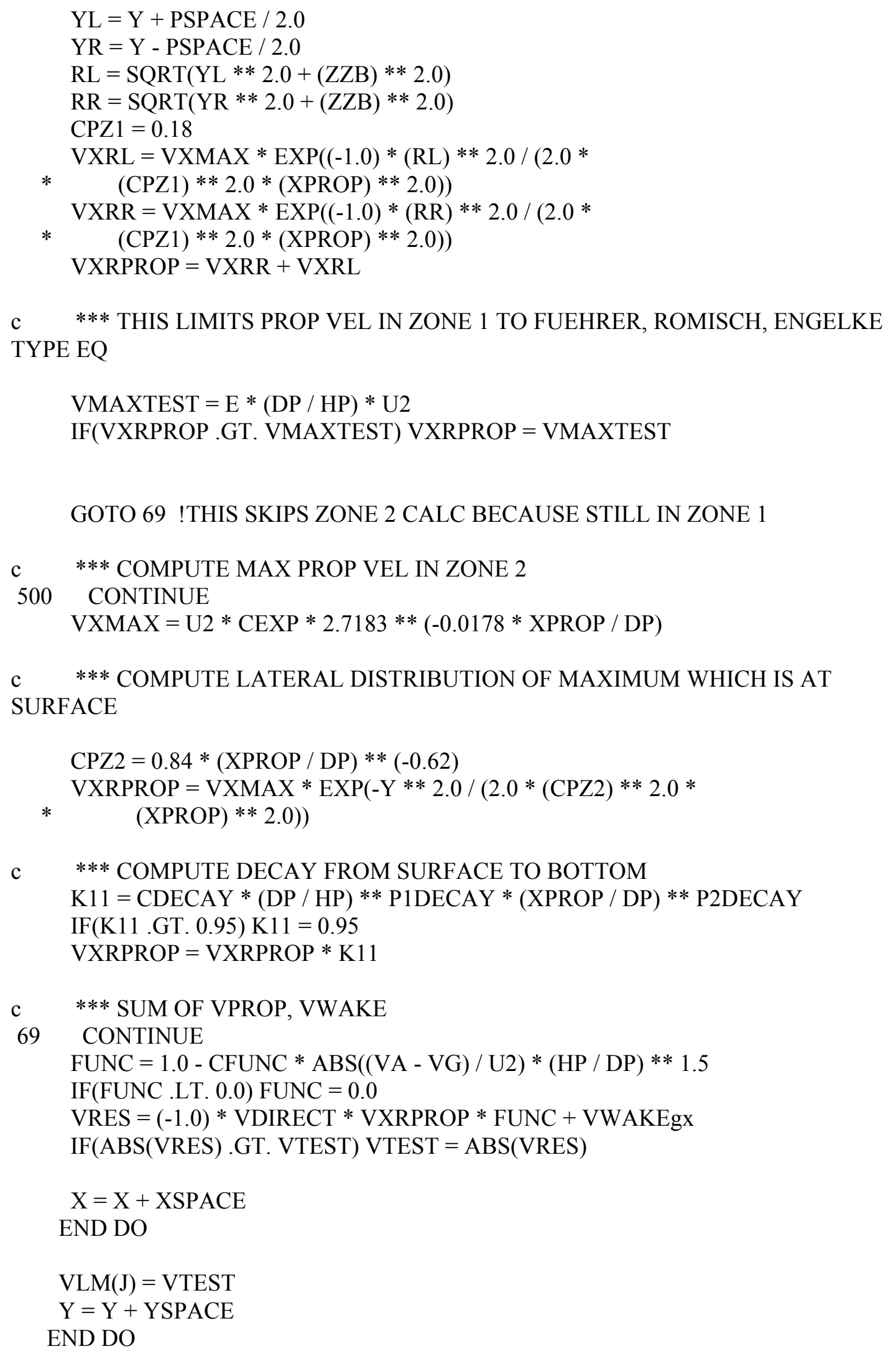


C

C

C

C

C

*** END ITERATION LOOP ON X AND Y

*** OUTPUT

PRINT "CL DIST, M MAX VEL CHANGE, M/SEC"

FOR K $=1$ TO NUMY

$\mathrm{YY}(1)=0$

PRINT YY(K), VLM(K)

NEXT K

PRINT "TOTAL INSTALLED HORSEPOWER $(=1.2 *$ APPLIED POWER $)=$ ", TIHP

80 CONTINUE

$\mathrm{C}==$
$\mathrm{C}==$ Start of Propeller Jet Velocities from Visual Basic Code $==$
$\mathrm{C}===============================+=======================$

DO $\mathrm{i}=$ LeftBankPnt, $($ TowLocPnt-1)

Distance $(\mathrm{i})=$ Distance $(\mathrm{i}) *(-1.0)$

END DO

NBNum $=$ RightNear - LeftNear +2.0

NBIncVal $=($ DrawDown $($ RightNear+1) - DrawDown $($ LeftNear-1) $) /$ NBNum

DO $\mathrm{i}=$ LeftNear, RightNear

DrawDown(i) $=$ DrawDown $($ i-1 $)+$ NBIncVal

END DO

NBNum $=$ RightNear - LeftNear +2.0

NBIncVal $=($ VelChange $($ RightNear+1 $)-$ VelChange $($ LeftNear-1) $) /$ NBNum

DO $\mathrm{i}=$ LeftNear, RightNear

VelChange $(\mathrm{i})=$ VelChange $(\mathrm{i}-1)+$ NBIncVal

END DO

IF(VLM(1) .GT. VelChange(TowLocPnt))

* VelChange $($ TowLocPnt $)=$ VLM $(1)$

IF(VLM(2) .GT. VelChange(TowLocPnt-1))

* VelChange(TowLocPnt-1) $=\operatorname{VLM}(2)$

IF(VLM(2) .GT. VelChange(TowLocPnt+1))

$*$ VelChange $($ TowLocPnt +1$)=\operatorname{VLM}(2)$

c IF(VelChange(TowLocPnt-1).LT. VelChange(LeftNear-1))

c * VelChange $($ TowLocPnt-1) $=$ VelChange $($ LeftNear-1)

c IF(VelChange(TowLocPnt+1) .LT. VelChange(RightNear+1))

c *VelChange $($ TowLocPnt +1$)=$ VelChange $($ RightNear +1$)$

c IF(VelChange(TowLocPnt).LT. VelChange(TowLocPnt-1))

c $\quad *$ VelChange $($ TowLocPnt $)=$ VelChange $($ TowLocPnt- 1$)$

c IF(VelChange(TowLocPnt).LT. VelChange(TowLocPnt+1))

c $\quad *$ VelChange $($ TowLocPnt $)=$ VelChange $($ TowLocPnt +1$)$

$\mathrm{c}^{* * * * *}$ Interpolation from Tow RVel to Original NAVEFF RVels ***** 
c

c

c

c

c

c

c

c

c

c

c

c

c

c

c

c

c

c

c

c

c

c

c

$\mathrm{C}==\quad$ Initialize Variables and Call Scour Routines $=$

$\mathrm{C}==$

IF(KOLocId .EQ. 1) AKO = "k" ! kort or open IF(KOLocId .EQ. 2) AKO = "o" ! kort or open

$\mathrm{C} \quad \mathrm{DP}=2.74 \quad$ ! propeller diameter $\operatorname{vg}=($ Velocity $($ VelLocId $) *$ Direction $)+$ AveVel $!$ vessel speed relative to ground DIRECT $=$ Direction $\quad !-1=$ upbound,$+1=$ downbound barbeam $=$ TowSize (SizeLocId,1) ! total width of barges DRAFTc $=$ Draft(DraftLocId) $\quad$ ! draft of barges barlen $=$ TowSize(SizeLocId,2) ! total length of barges tbl $=52.0 \quad$ ! length of towboat- keep constant for all tows

C THRUST $=300000.0$ ! thrust for each props in newtons $\mathrm{VNU}=.000001 \quad$ ! kinematic viscosity- fix for all conditions?????

Ylamb $=.3 \quad$ ! porosity of sediment- fix for all ?????????????? 


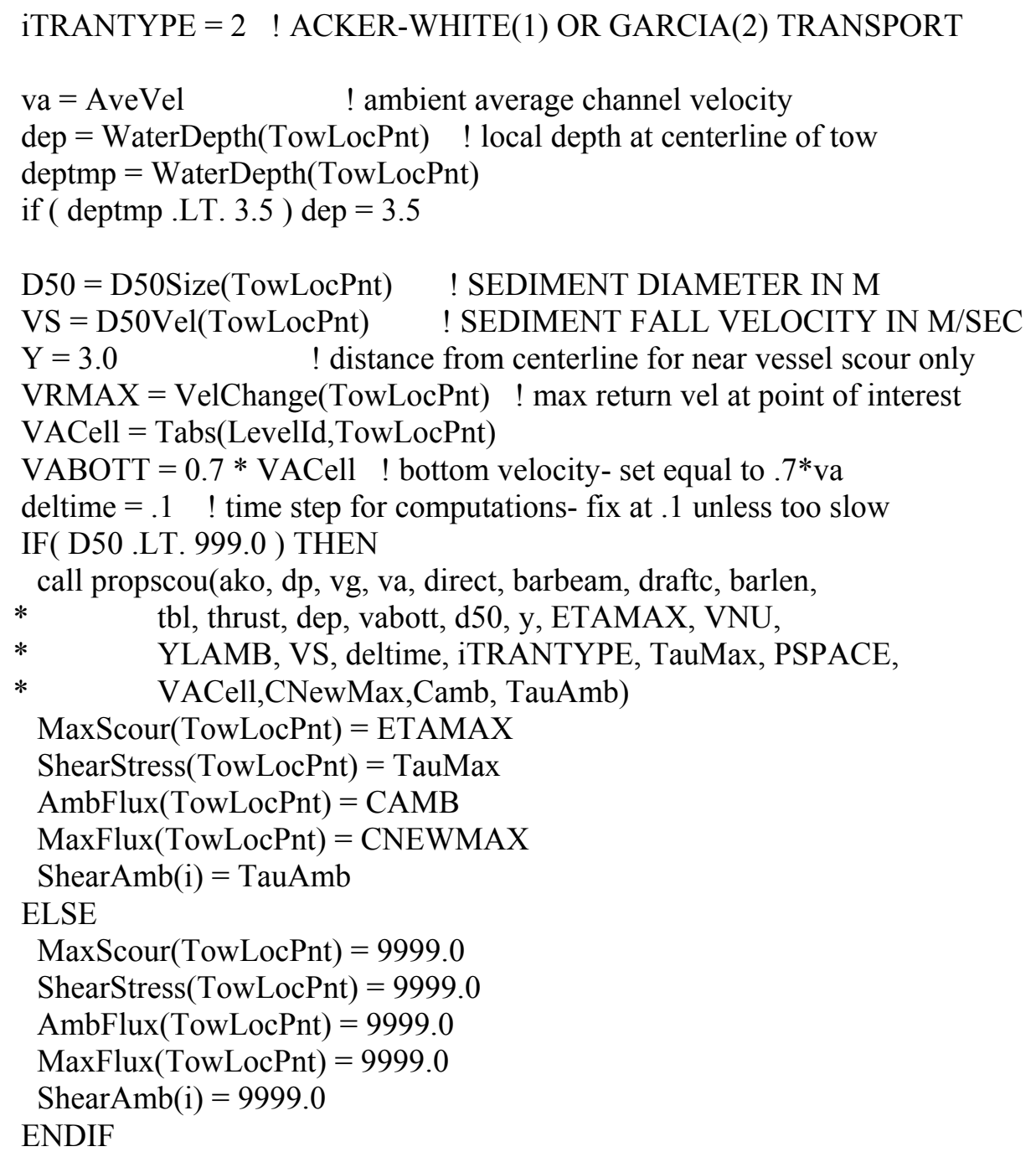




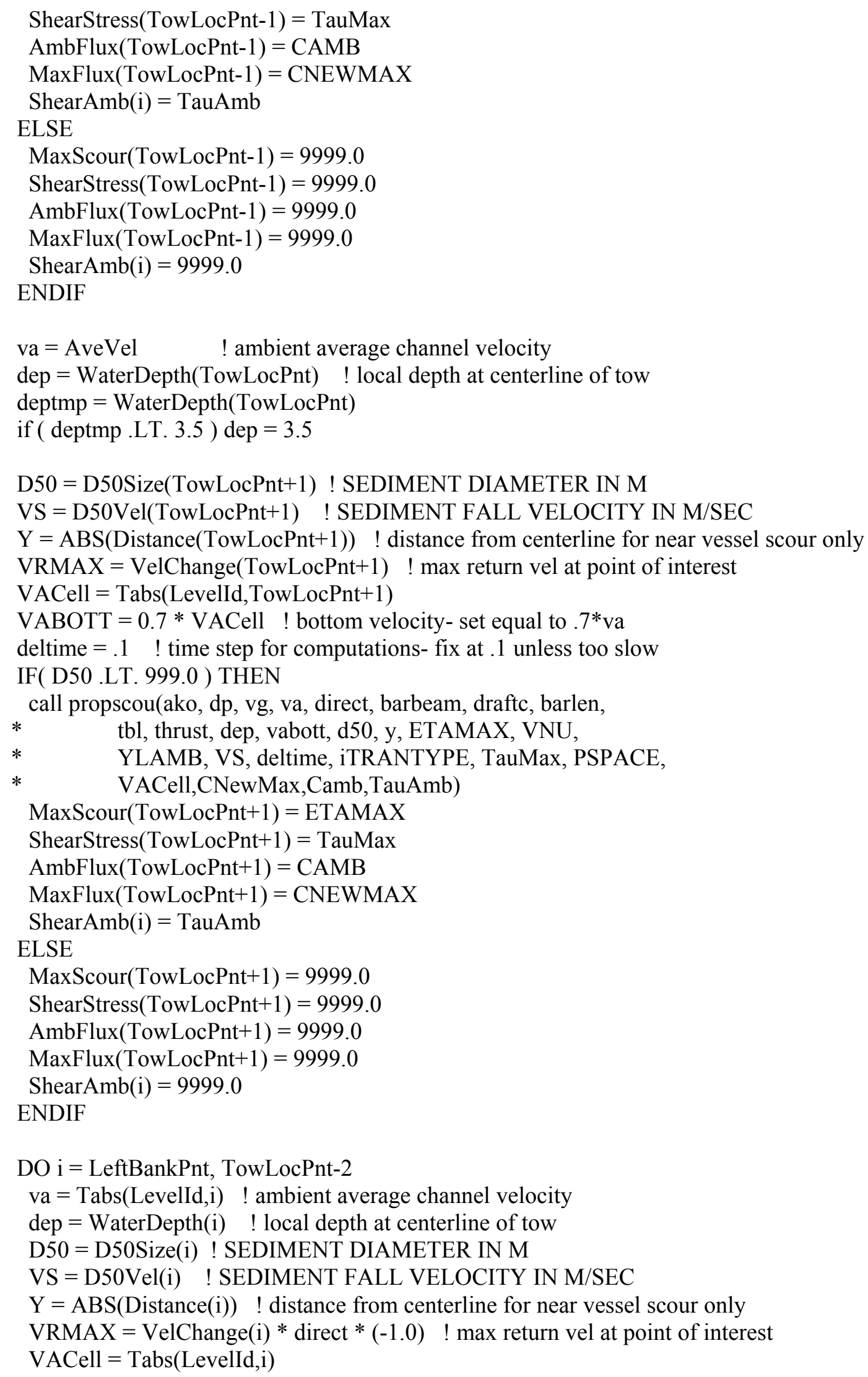




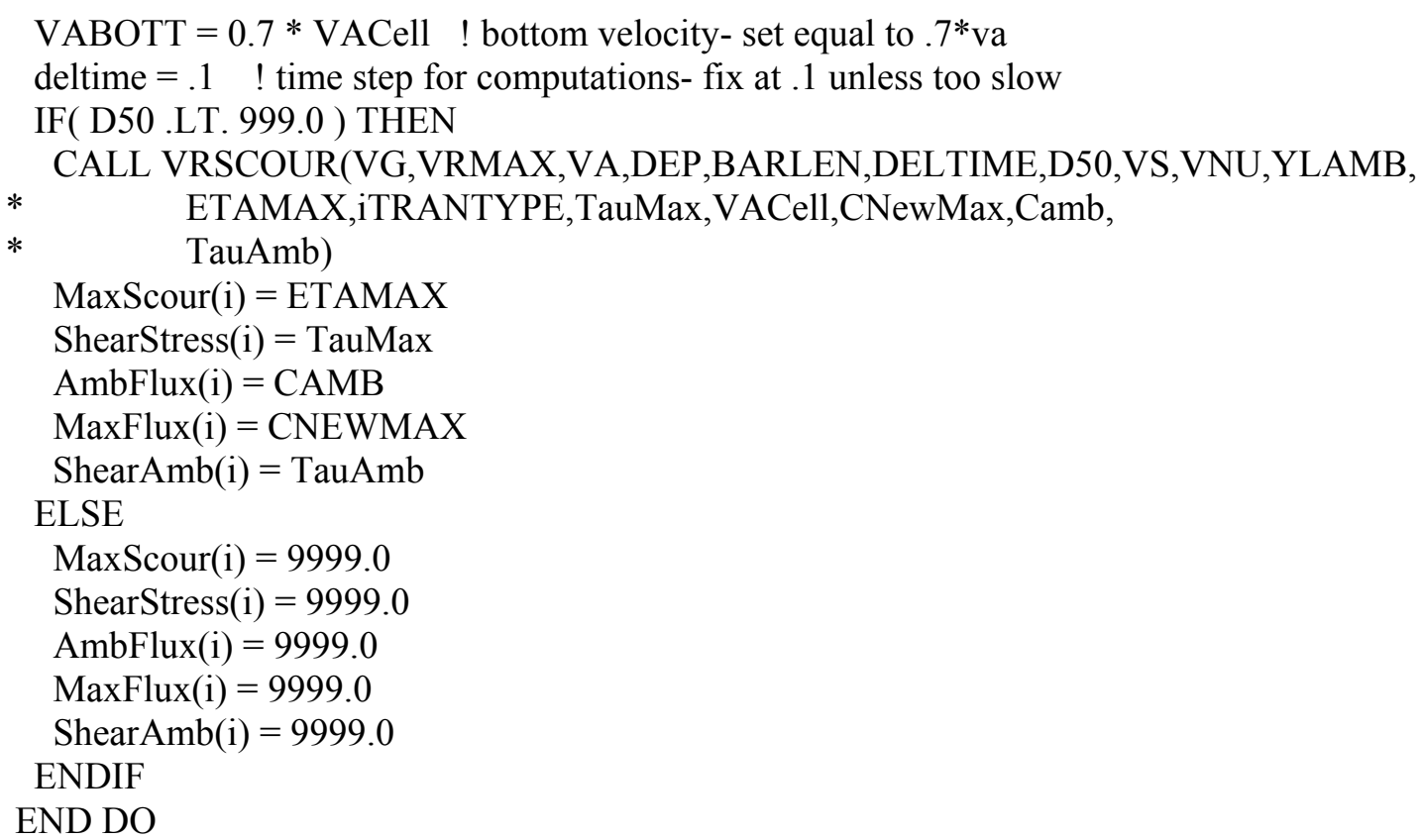




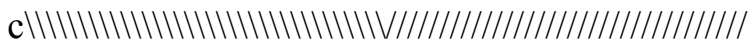

$\mathrm{c} * * * * * * * * * * * * * * * * * * * * * * * * * * * * * * * * * * * * * * * * * * * * * * * * * * * * * * * * * * * * * * * * * * * *$

$\mathrm{C}==$

$\mathrm{C}==\quad$ Initialize Variables and Call Scour Routines $=$

C $======================================================$

DO i = LeftBankPnt, RightBankPnt

IF(VelChange(i) .GE. 10.0) GOTO 855

IF(DrawDown(i) .GE. 2.0) GOTO 856

IF(Distance(i) .GE. 10000.0) GOTO 857

IF(SecWaveHgt(i) .GE. 0.42) GOTO 858

END DO

c WRITE $(8,880)$ ErrorCode,RiverMile,DirLoc,VelLoc,SizeLoc,

c * DraftLoc,KOLoc,PoolLevel,TowLoc,DirLoc,VelLoc,

c * SizeLoc,DraftLoc,KOLoc,PoolLevel,TowLoc,ATOTAL,

c * ALEFT,BTOTAL,BLEFT,B,L,D,V,VAM,

c * MaxDepth,HSave1,HSave2,U1,ZT

DO i = LeftBankPnt, RightBankPnt

WRITE(9, 950) RiverMile, DirLoc, VelLoc, SizeLoc, DraftLoc,

* KOLoc, PoolLevel, TowLoc, DirLoc, VelLoc,

* SizeLoc, DraftLoc, KOLoc, PoolLevel, TowLoc,

* $\quad$ BinLabel(i), WaterDepth(i), VelChange(i),

* DrawDown(i), Distance(i), SecWaveHgt(i),

* $\quad$ MaxScour(i), ShearStress(i), AmbFlux(i),

* MaxFlux(i), ShearAmb(i)

END DO

950 format(f5.1,,',a1,,',,a1,,',,a1,,',,a1,,',,a1,',', a1,,',',

* a1,,',,a1,a1,a1,a1,a1,,',, a1,a1,,',,a10,',,'f5.2,',',

* $\quad$ f6.3,',',f6.3,',',f8.1,',',f6.3,',',f9.4,',',f9.4,',',

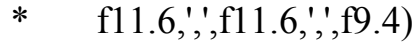

GOTO 99

862 CONTINUE

ErrorCode $=$ 'RBnk' !Could not find Right Bank

GOTO 850

861 CONTINUE

ErrorCode $=$ 'LBnk' !Could not find Left Bank

GOTO 850

860 CONTINUE

ErrorCode $=$ 'TLoc' ! Could not find Tow Loc

GOTO 850

859 CONTINUE

ErrorCode $=$ 'Tran' !Could not find Transect

GOTO 850

858 CONTINUE 


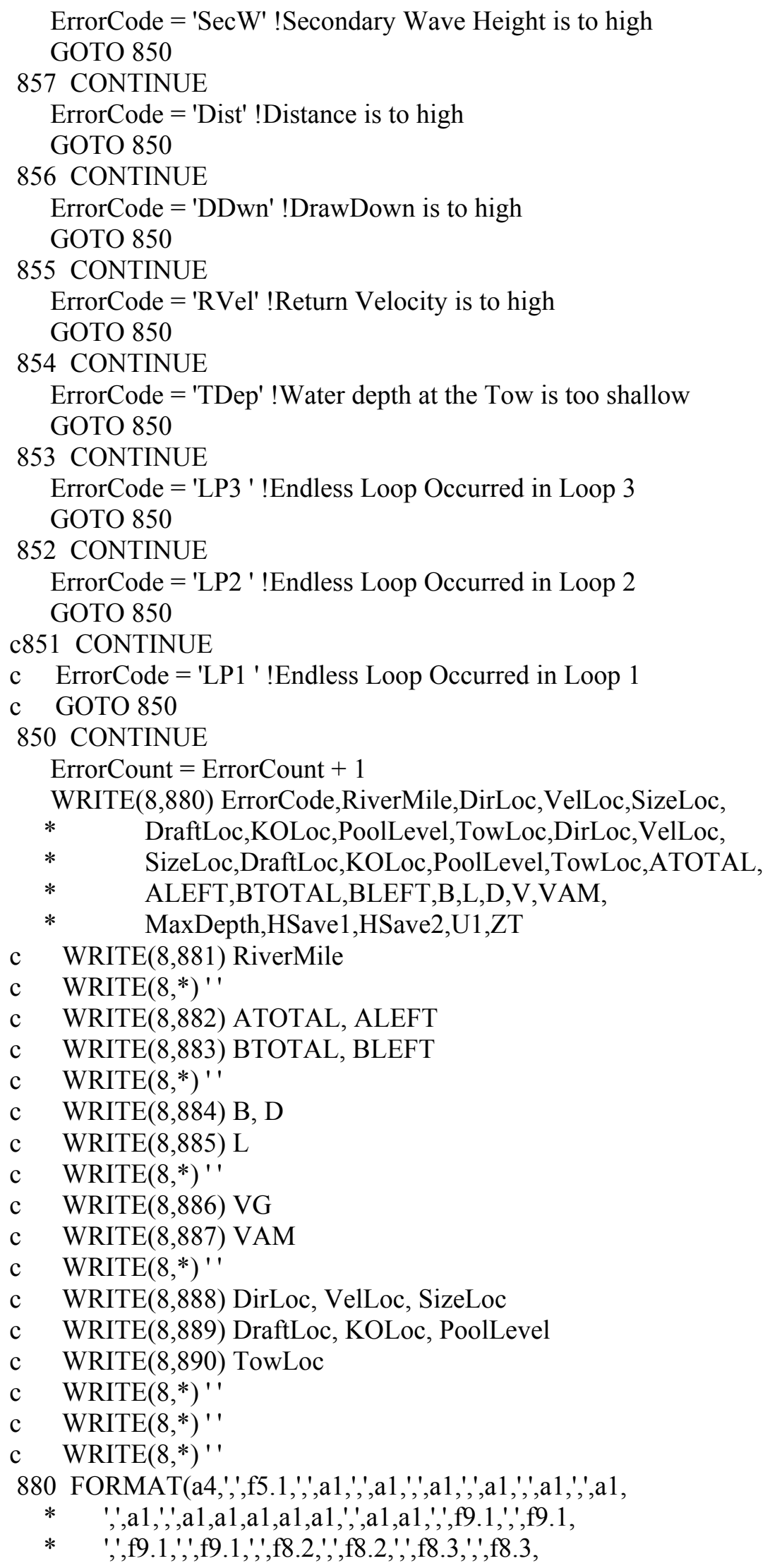




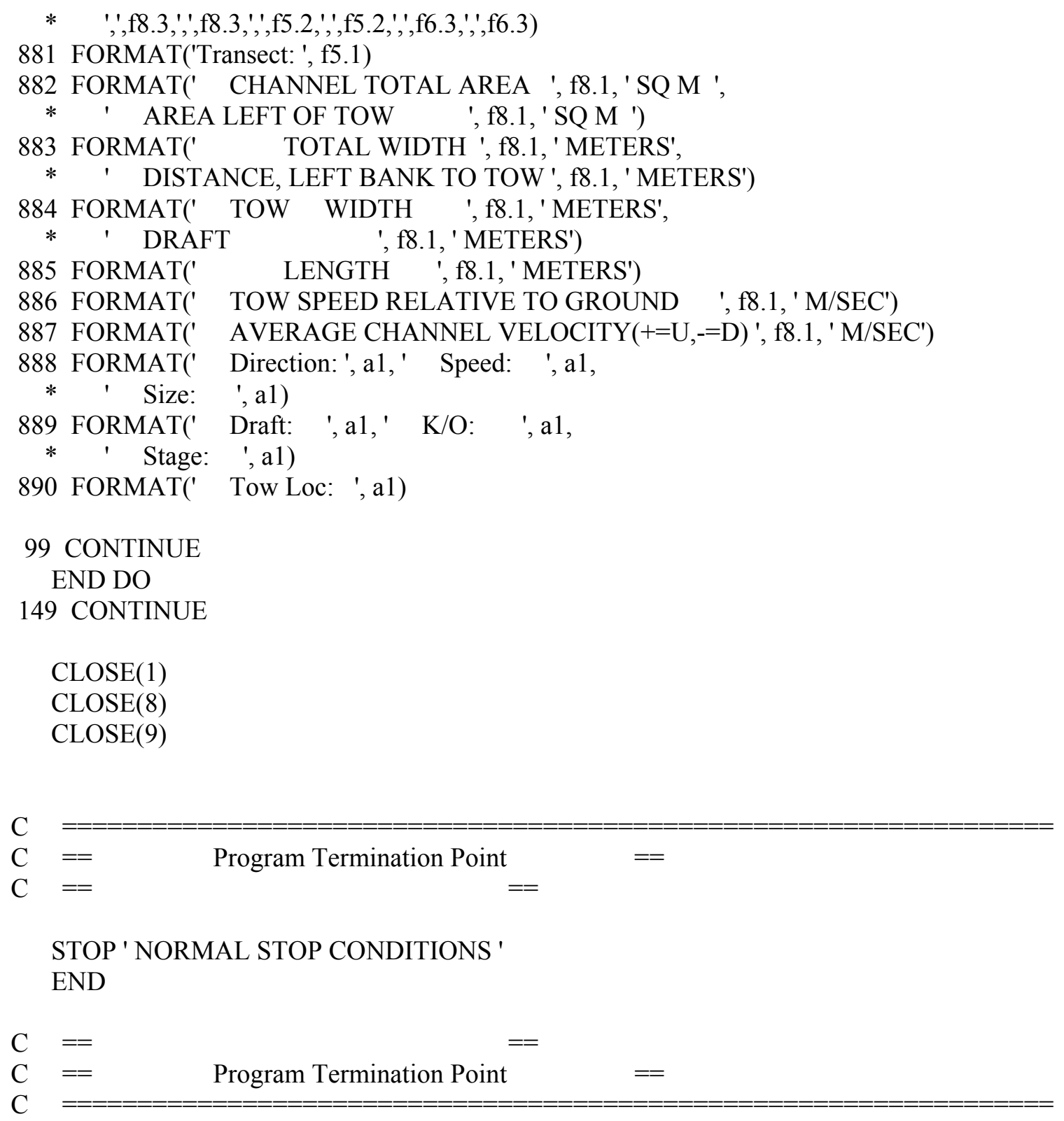

subroutine vrscour(vg,vrmax,va,dep, barlen,deltime,d50,vs,vnu,

* \& Ylamb, ETAMAX, iTRANTYPE,TauMax,VACell,CNewMax,Camb, TauAmb)

* SUBROUTINE VRSCOUR DEFINES THE SCOUR FOR THE ZONE AWAY FROM THE VESSEL

*

DIMension vrhis $(19,2)$

$*$

* DIMENSIONLESS RETURN VELOCITY DISTRIBUTION FROM KAMPSVILLE REPORT $*$ 
$\operatorname{vrhis}(1,1)=0$

$\operatorname{vrhis}(1,2)=0$

$\operatorname{vrhis}(2,1)=.2$

$\operatorname{vrhis}(2,2)=.02$

$\operatorname{vrhis}(3,1)=.4$

$\operatorname{vrhis}(3,2)=.1$

$\operatorname{vrhis}(4,1)=.6$

$\operatorname{vrhis}(4,2)=.21$

$\operatorname{vrhis}(5,1)=.7$

$\operatorname{vrhis}(5,2)=.34$

$\operatorname{vrhis}(6,1)=.8$

$\operatorname{vrhis}(6,2)=.5$

$\operatorname{vrhis}(7,1)=.9$

$\operatorname{vrhis}(7,2)=.64$

$\operatorname{vrhis}(8,1)=1.0$

$\operatorname{vrhis}(8,2)=.77$

$\operatorname{vrhis}(9,1)=1.1$

$\operatorname{vrhis}(9,2)=.83$

$\operatorname{vrhis}(10,1)=1.2$

$\operatorname{vrhis}(10,2)=.86$

$\operatorname{vrhis}(11,1)=1.3$

$\operatorname{vrhis}(11,2)=.9$

$\operatorname{vrhis}(12,1)=1.4$

$\operatorname{vrhis}(12,2)=.95$

$\operatorname{vrhis}(13,1)=1.5$

$\operatorname{vrhis}(13,2)=1.0$

$\operatorname{vrhis}(14,1)=1.6$

$\operatorname{vrhis}(14,2)=.92$

$\operatorname{vrhis}(15,1)=1.8$

$\operatorname{vrhis}(15,2)=.65$

$\operatorname{vrhis}(16,1)=2$

$\operatorname{vrhis}(16,2)=.36$

$\operatorname{vrhis}(17,1)=2.2$

$\operatorname{vrhis}(17,2)=.07$

$\operatorname{vrhis}(18,1)=2.3$

$\operatorname{vrhis}(18,2)=.001$

$\operatorname{vrhis}(19,1)=50$

$\operatorname{vrhis}(19,2)=0$

*

* INITIALIZE VARIABLES

$*$

c $\quad$ GRAV $=9.805$

TauMax $=0.0$

CNewMax $=0.0$

rho $=1000$

cold $=0$

$\mathrm{ETA}=0.0$

ETAMAX $=0.0$

TIMSCOUR $=0$

timbarge $=$ barlen $/$ ABS(vg) 


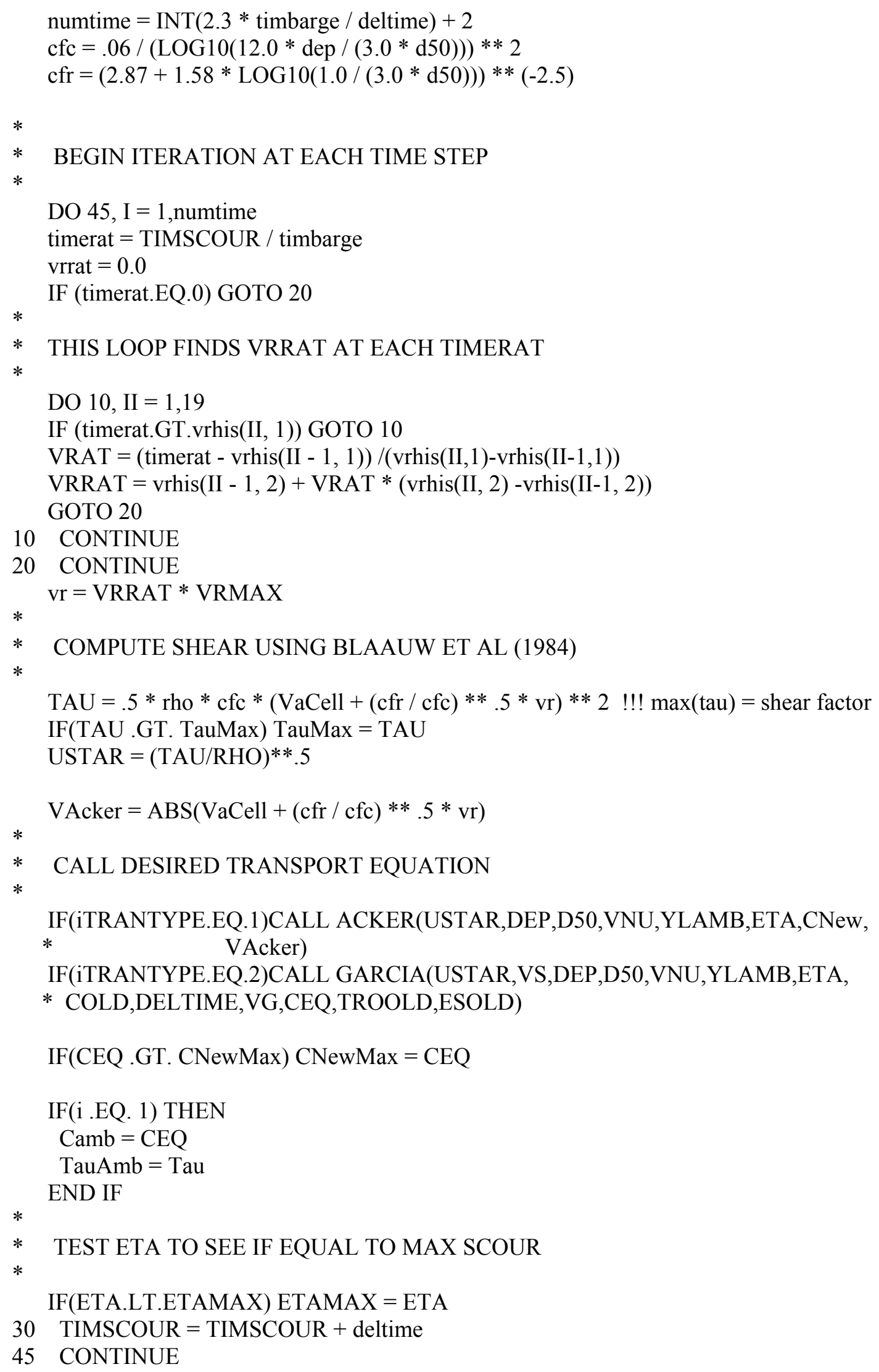


*

subroutine propscou(ako, dp, vg, va, direct, barbeam, draft, \& barlen,tbl,thrust,dep, vabott, d50,y,ETAMAX,VNU,YLAMB,VS, \& deltime,iTRANTYPE,TauMax,PSPACE,VACell,CNewMax,Camb,TauAmb)

$*$

* SUBROUTINE PROPSCOU DEFINES SCOUR BENEATH VESSEL

*

DIMension taup $(12,5), \operatorname{taub}(9,2)$

*

$*$

*

$\operatorname{taub}(1,1)=0.0$

$\operatorname{taub}(1,2)=-1.17$

$\operatorname{taub}(2,1)=.25$

$\operatorname{taub}(2,2)=-.73$

$\operatorname{taub}(3,1)=.5$

$\operatorname{taub}(3,2)=-.51$

$\operatorname{taub}(4,1)=.75$

$\operatorname{taub}(4,2)=-.33$

$\operatorname{taub}(5,1)=1.0$

$\operatorname{taub}(5,2)=0.0$

$\operatorname{taub}(6,1)=.75$

$\operatorname{taub}(6,2)=.37$

$\operatorname{taub}(7,1)=.5$

$\operatorname{taub}(7,2)=.67$

$\operatorname{taub}(8,1)=.25$

$\operatorname{taub}(8,2)=1.41$

$\operatorname{taub}(9,1)=0.0$

$\operatorname{taub}(9,2)=3.41$

*

* DIMENSIONLESS array for propeller SHEAR DISTRIBUTION

$*$

$\operatorname{taup}(1,1)=0.0$

$\operatorname{taup}(1,2)=-6.0$

$\operatorname{taup}(1,3)=-25.0$

$\operatorname{taup}(1,4)=-50.0$

$\operatorname{taup}(1,5)=0.0$

$\operatorname{taup}(2,1)=.1$

$\operatorname{taup}(2,2)=-3.3$

$\operatorname{taup}(2,3)=-15.0$

$\operatorname{taup}(2,4)=-30.0$

$\operatorname{taup}(2,5)=0.0$

$\operatorname{taup}(3,1)=.25$

$\operatorname{taup}(3,2)=-2.1$ 


$$
\begin{aligned}
& \operatorname{taup}(3,3)=-6.4 \\
& \operatorname{taup}(3,4)=-11 \\
& \operatorname{taup}(3,5)=0 \\
& \operatorname{taup}(4,1)=.5 \\
& \operatorname{taup}(4,2)=-1.4 \\
& \operatorname{taup}(4,3)=-1.5 \\
& \operatorname{taup}(4,4)=-2.7 \\
& \operatorname{taup}(4,5)=0 \\
& \operatorname{taup}(5,1)=.75 \\
& \operatorname{taup}(5,2)=-.54 \\
& \operatorname{taup}(5,3)=-.6 \\
& \operatorname{taup}(5,4)=-.7 \\
& \operatorname{taup}(5,5)=0 \\
& \operatorname{taup}(6,1)=1.0 \\
& \operatorname{taup}(6,2)=0 \\
& \operatorname{taup}(6,3)=0 \\
& \operatorname{taup}(6,4)=0 \\
& \operatorname{taup}(6,5)=0 \\
& \operatorname{taup}(7,1)=.75 \\
& \operatorname{taup}(7,2)=.6 \\
& \operatorname{taup}(7,3)=1.3 \\
& \operatorname{taup}(7,4)=1.7 \\
& \operatorname{taup}(7,5)=0 \\
& \operatorname{taup}(8,1)=.5 \\
& \operatorname{taup}(8,2)=2.2 \\
& \operatorname{taup}(8,3)=9.2 \\
& \operatorname{taup}(8,4)=15 \\
& \operatorname{taup}(8,5)=0 \\
& \operatorname{taup}(9,1)=.25 \\
& \operatorname{taup}(9,2)=11 \\
& \operatorname{taup}(9,3)=37 \\
& \operatorname{taup}(9,4)=60 \\
& \operatorname{taup}(9,5)=0 \\
& \operatorname{taup}(10,1)=.1 \\
& \operatorname{taup}(10,2)=23 \\
& \operatorname{taup}(10,3)=93 \\
& \operatorname{taup}(10,4)=125 \\
& \operatorname{taup}(10,5)=0 \\
& \operatorname{taup}(11,1)=.05 \\
& \operatorname{taup}(11,2)=75 \\
& \operatorname{taup}(11,3)=153 \\
& \operatorname{taup}(11,4)=175 \\
& \operatorname{taup}(11,5)=0 \\
& \operatorname{taup}(12,1)=0 \\
& \operatorname{taup}(12,2)=230 \\
& \operatorname{taup}(12,3)=230 \\
& \operatorname{taup}(12,4)=230 \\
& \operatorname{taup}(12,5)=0
\end{aligned}
$$

* BEGIN INPUT 
$*$

* OPEN(3,"temp.dat", FORM ='FORMATTED', STATUS = 'UNKNOWN')

IF(AKO.EQ.'k') AKO = 'K'

$\mathrm{IF}\left(\mathrm{AKO} . \mathrm{EQ} . \mathrm{o}^{\prime}\right) \mathrm{AKO}=$ 'O'

TauMax $=0.0$

$\mathrm{CNewMax}=0.0$

cold $=0$

c $\quad \mathrm{vg}=\mathrm{vg} *$ DIRECT

c PSPACE $=6 \quad$ !!! FIX

c $\quad$ GRAV $=9.805$

SETBACK $=5$

c $\quad$ THRUST $=$ THRUST $/ 2$

$\mathrm{hp}=\operatorname{dep}-\mathrm{DP} / 2$

* TAUFAC IS THE RATIO USED TO ADJUST PHYSICAL MODEL VALUES FROM THE

* SMOOTH BOUNDARY TO THE ROUGH BOUNDARY FOUND IN THE FIELD AND

TO

* ACCOUNT FOR THE GREATER TURBULENCE FOUND IN PROPELLER JETS

* COMPARED TO OPEN CHANNEL FLOW USED TO DEVELOP TRANSPORT EQUATIONS

*

taufac $=7.87 *(\mathrm{~d} 50) * *(.18)$

ETAMAX $=0.0$

$\mathrm{ETA}=0.0$

15 continue

$\mathrm{XSPc}=\mathrm{ABS}($ deltime $* \mathrm{vg})$

$\mathrm{XBEGIN}=0$

numx $=\operatorname{INT}(($ barlen + tbl $+.05 /$ D50 $) / \mathrm{XSPc})$

NUMY $=1$

rho $=999.8$

$\mathrm{CFCamb}=0.06 /($ LOG10 $(12.0 * \mathrm{DEP} /(3.0 * \mathrm{D} 50))) * * 2$

TAUAMB $=0.5 *$ RHO $*$ CFCAMB $*$ VACell $* * 2$

$\mathrm{USTAR}=(\mathrm{TAUAMB} / \mathrm{RHO}) * * 0.5$

VAcker $=$ VACell

CALL GARCIA(USTAR,VS,DEP,D50,VNU,YLAMB,ETA,

* COLD,DELTIME,VG,CEQ,TROOLD,ESOLD)

$\mathrm{CAMB}=\mathrm{CEQ}$

$*$

* SET KORT OR OPEN PARAMETERS

*

IF(AKO.EQ.'K') GO TO 12

$*$

* THIS SECTION FOR OPEN WHEEL

$\mathrm{D} 0=.71 * \mathrm{DP}$

$\mathrm{E}=.43$

$\mathrm{CFUNC}=.5$

GOTO 17 


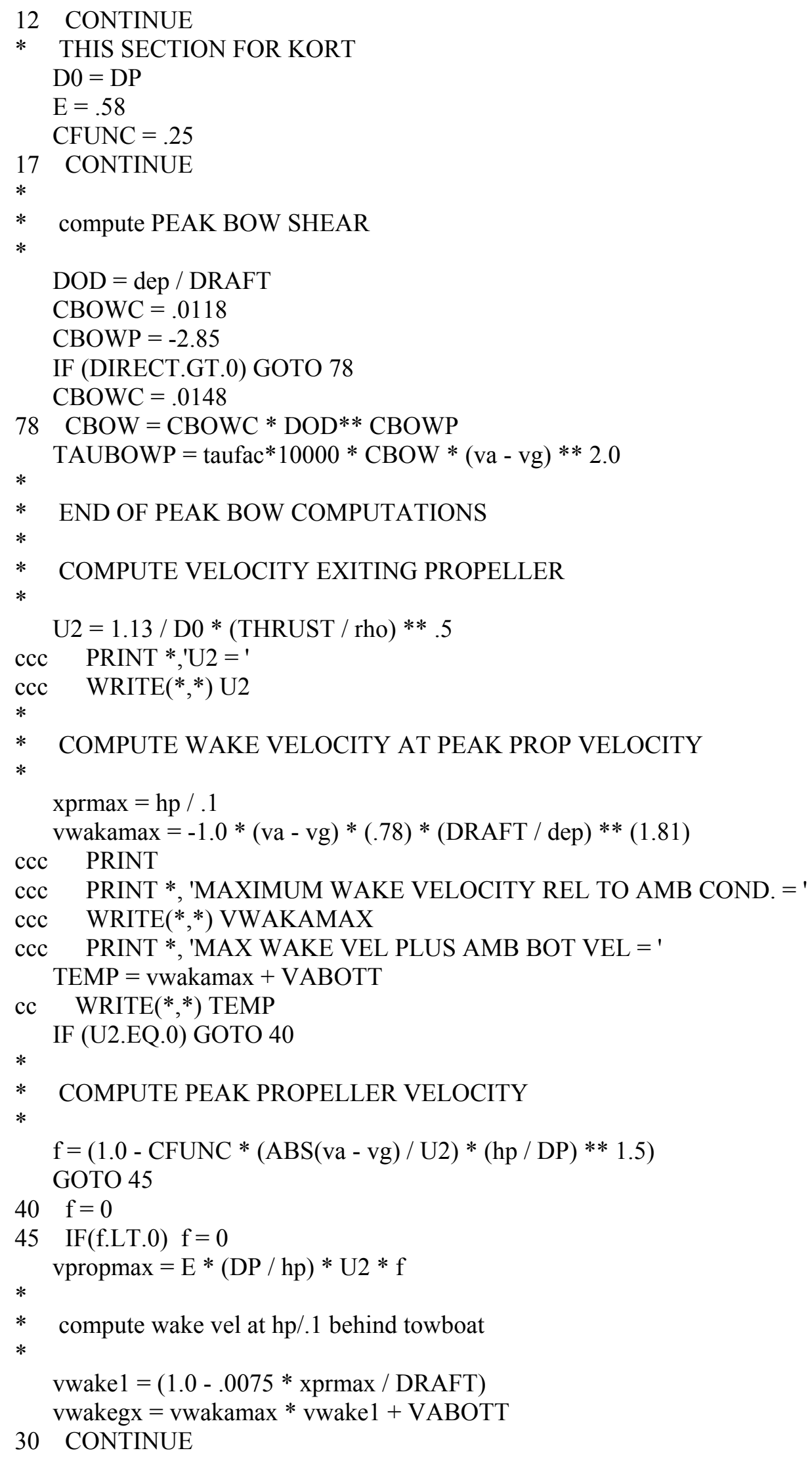




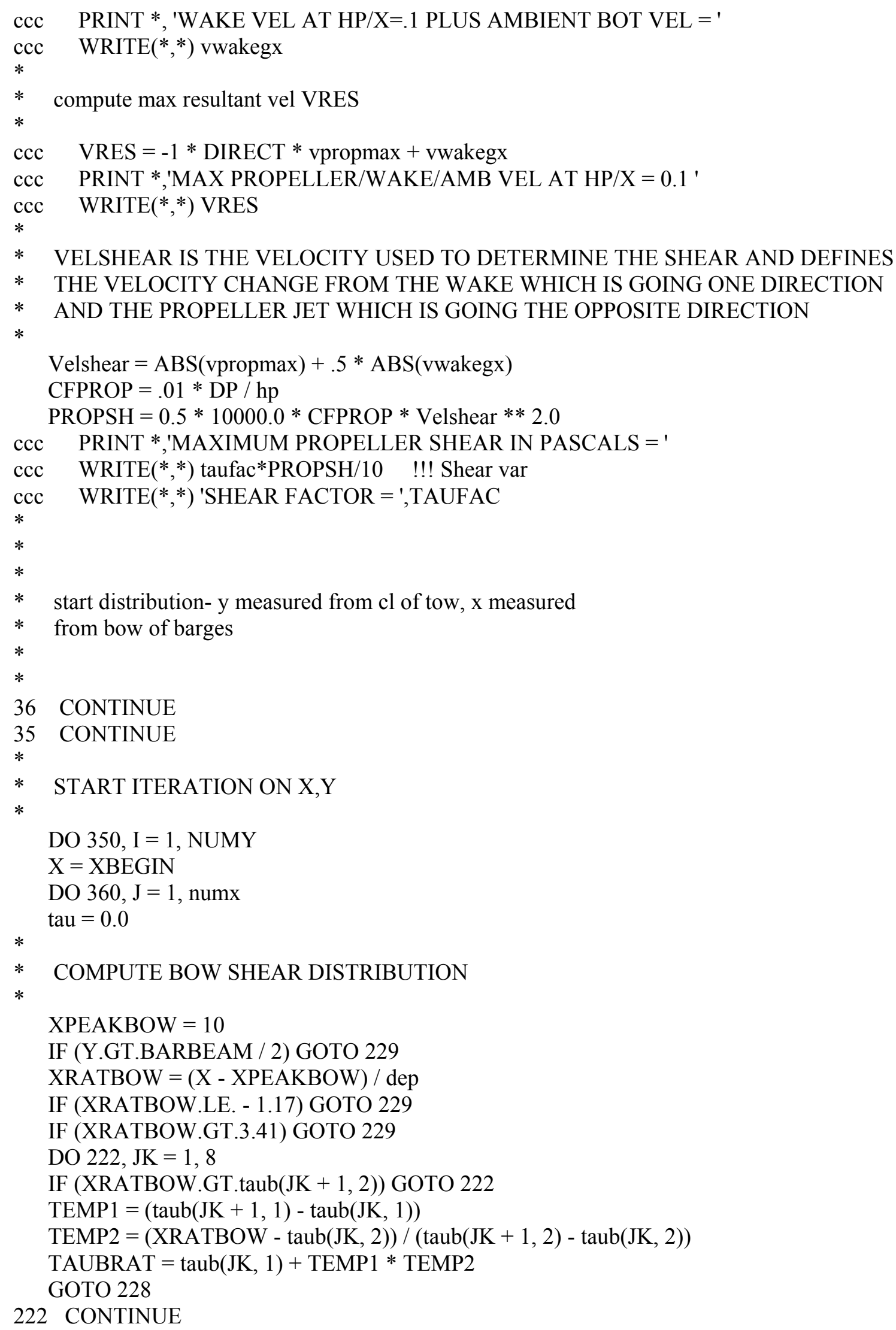




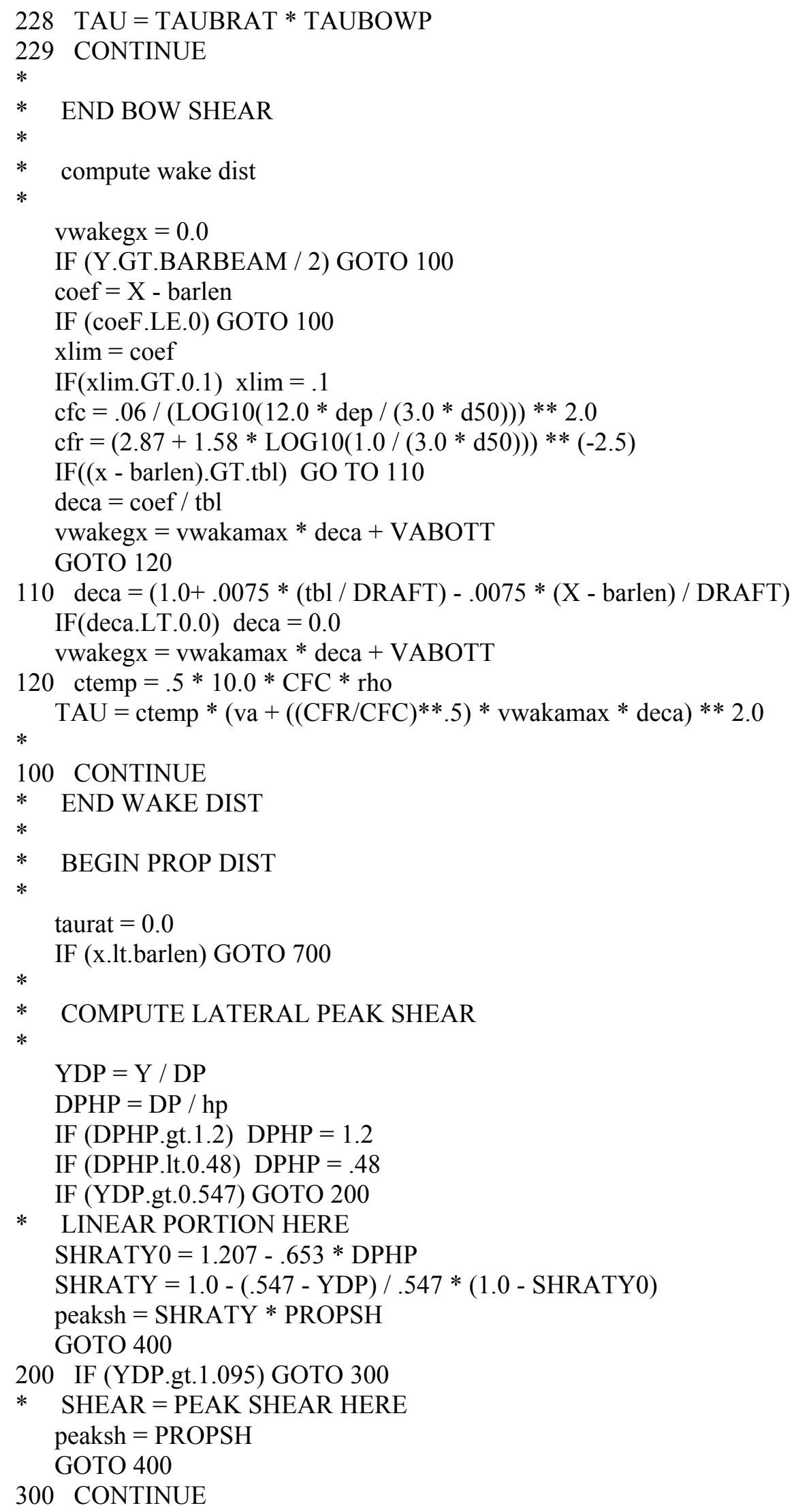


* EXPONENTIAL SHEAR HERE

$\mathrm{C} 5=.0221 * 2.7183 * *(3.14 * \mathrm{DPHP})$

SHRATY $=2.7183 * *((-1.0 * \mathrm{C} 5) *((\mathrm{Y}-\mathrm{PSPACE} / 2) / \mathrm{DP}) * * 2)$

peaksh $=$ SHRATY $*$ PROPSH

$*$

* END FINDING LATERAL PEAK SHEAR

400 CONTINUE

*

* COMPUTE LONGITUDINAL SHEAR FROM PEAK LATERAL SHEAR

$*$

$\mathrm{XPEAK}=$ barlen $+\mathrm{tbl}+\mathrm{hp} / .2-\mathrm{SETBACK}$

$\mathrm{XRAT}=(\mathrm{X}-\mathrm{XPEAK}) / \mathrm{hp}$

$*$

* PS IS ONLY USED TO SELECT WHICH LONGITUDINAL DISTRIBUTION TO USE

* ALL TAU IN THIS SECTION ARE IN DYNES/SQ CM AND HAVE NOT BEEN ADJUSTED

* BY TAUFAC. THIS IS NECESSARY TO FIT THE RANGES OF PS FOR FITTING THE

* DISTRIBUTIONS.

PS $=$ peaksh

IF (peaksh.gt.1000.0) PS $=1000.0$

IF (peaksh.lt.69.0) PS $=69.0$

IF (PS.It.215.0) GOTO 500

* THIS PART IS FOR PS FROM 1000-215

do $450, \mathrm{jj}=1,12$

TEMP $=(1000.0-\mathrm{PS}) / 785.0 *(\operatorname{taup}(\mathrm{JJ}, 3)-\operatorname{taup}(\mathrm{JJ}, 2))$

$\operatorname{taup}(\mathrm{JJ}, 5)=\operatorname{taup}(\mathrm{JJ}, 2)+\mathrm{TEMP}$

450 CONTINUE

GOTO 600

500 CONTINUE

* THIS PART FOR PS $<215$ TO 69

DO $550, \mathrm{kk}=1,12$

TEMP $=(215.0-\mathrm{PS}) / 146.0 *(\operatorname{taup}(\mathrm{KK}, 4)-\operatorname{taup}(\mathrm{KK}, 3))$

$\operatorname{taup}(\mathrm{KK}, 5)=\operatorname{taup}(\mathrm{KK}, 3)+\mathrm{TEMP}$

550 CONTINUE

600 IF (XRAT.LE.taup $(1,5))$ GOTO 900

IF (XRAT.GE.230) GOTO 900

DO $650 \mathrm{k}=1,11$

IF (XRAT.GT.taup $(\mathrm{k}+1,5))$ GOTO 650

TEMP1 $=(\operatorname{taup}(\mathrm{k}+1,1)-\operatorname{taup}(\mathrm{k}, 1))$

TEMP2 $=$ TEMP1 $*($ XRAT $-\operatorname{taup}(\mathrm{k}, 5)) /(\operatorname{taup}(\mathrm{k}+1,5)-\operatorname{taup}(\mathrm{k}, 5))$

taurat $=\operatorname{taup}(\mathrm{k}, 1)+$ TEMP2

GOTO 700

650 CONTINUE

*

* END LONGITUDINAL DISTRIBUTION

$*$

* SET TAU to MAX OF PROP OR WAKE SHEAR AND ADJUST BY TAUFAC

* (TAU STILL IN DYNES/SQ CM) 


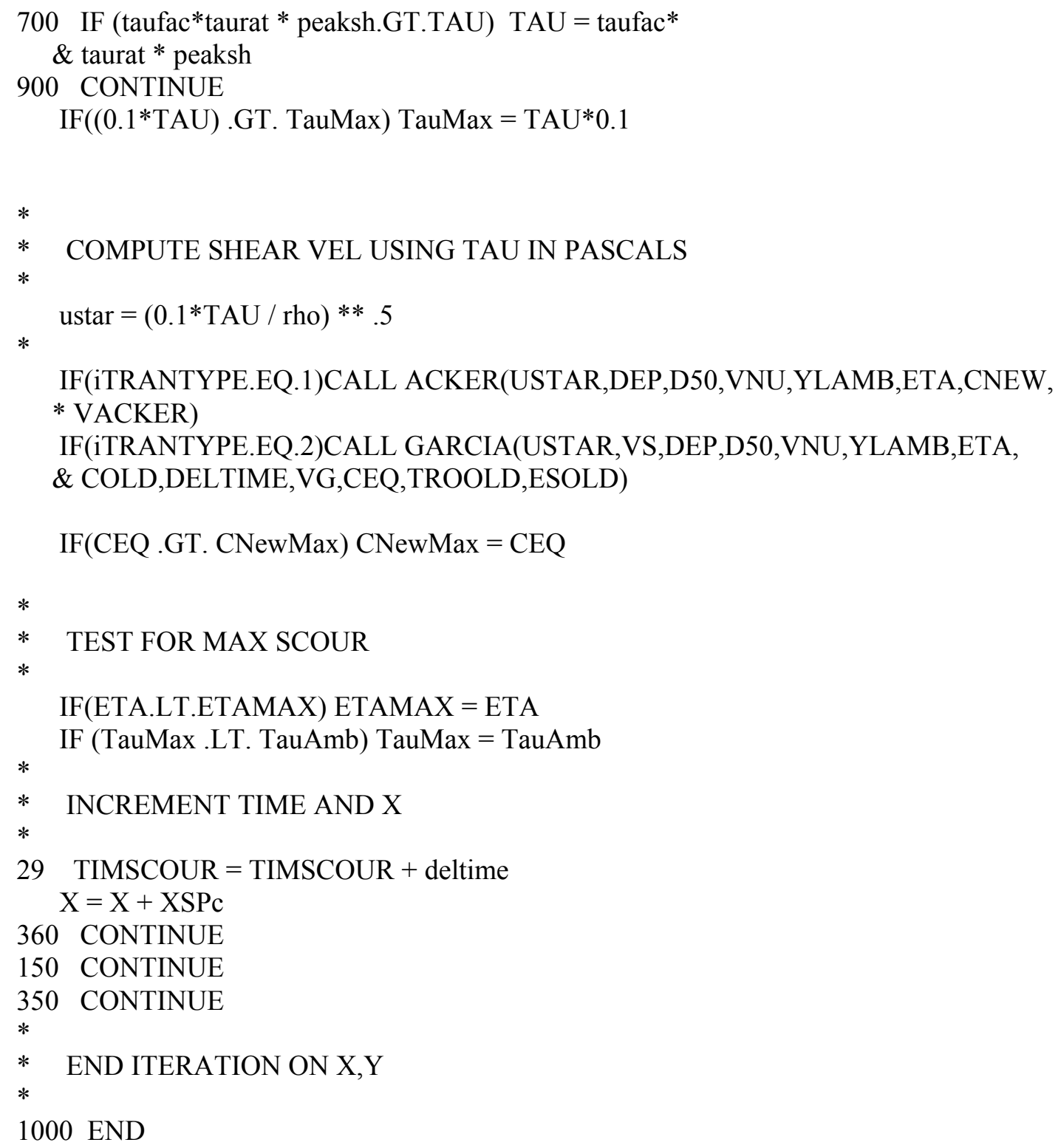


$\mathrm{EN}=0.0$

$\mathrm{A}=.17$

$\mathrm{EM}=1.78$

$\mathrm{C}=.025$

GOTO 23

22 CONTINUE

* TRANSITION PARAMETERS

$\mathrm{EN}=1-.56^{*}$ LOG10(dgr)

$\mathrm{A}=.23 /(\mathrm{dgr}) * * .5+.14$

$\mathrm{EM}=6.83 / \mathrm{dgr}+1.67$

$\mathrm{Cc}=2.79 *$ LOG10(dgr) $-0.98 *($ LOG10(dgr $)) * * 2-3.46$

$\mathrm{C}=10 * \mathrm{Cc}$

23 CONTINUE

* SEDIMENT MOBILITY

$\mathrm{FGR} 1=(\mathrm{GRAV} * \mathrm{~d} 50 * 1.65) * * .5$

FGR $11=$ ustar $* *$ EN $/$ FGR 1

$\mathrm{FGR} 2=10 *$ dep $/ \mathrm{d} 50$

FGR21 $=5.675 *$ LOG10(FGR2)

FGR22 $=($ vacker $/$ FGR21 $) * *(1-\mathrm{EN})$

$\mathrm{FGR}=\mathrm{FGR} 11 *$ FGR22

* TEST FOR ZERO TRANSPORT

$\mathrm{ZQS}=\mathrm{FGR} / \mathrm{A}$

IF(ZQS.LE.1.0) GO TO 25

$\mathrm{GGR}=\mathrm{C} *(\mathrm{FGR} / \mathrm{A}-1) * * \mathrm{EM}$

* $\quad$ cnew $=$ SED FLUX IN PARTS/PART by volume

cnew $=($ GGR $*$ d50) $/($ dep $*($ ustar $/$ vacker $) * *$ EN $)$

*

* $\quad$ LIMIT CNEW TO 0.3

$*$

if(cnew.gt.0.3) cnew $=0.3$

GOTO 26

$25 \mathrm{CNEW}=0$

26 CONTINUE

$*$

* EXNER EQ

$*$

ETA $=-1.0 *($ dep $*$ cnew $) /(1-$ Ylamb $)$

*

RETURN

END

$*$

SUBROUTINE GARCIA(USTAR,VS,DEP,D50,VNU,YLAMB,ETA,COLD,DELTIME,VG,

* CEQ,TROOLD,ESOLD)

* GARCIA FUNCTION FOR SCOUR

c WRITE $(8, *)$ VS,COLD,DELTIME,VG

GRAV $=9.805$

$\mathrm{RHO}=1000.0$

IF (ustar.EQ.0.0) GOTO 25

TAU $=$ RHO $*$ USTAR $* * 2.0$ 


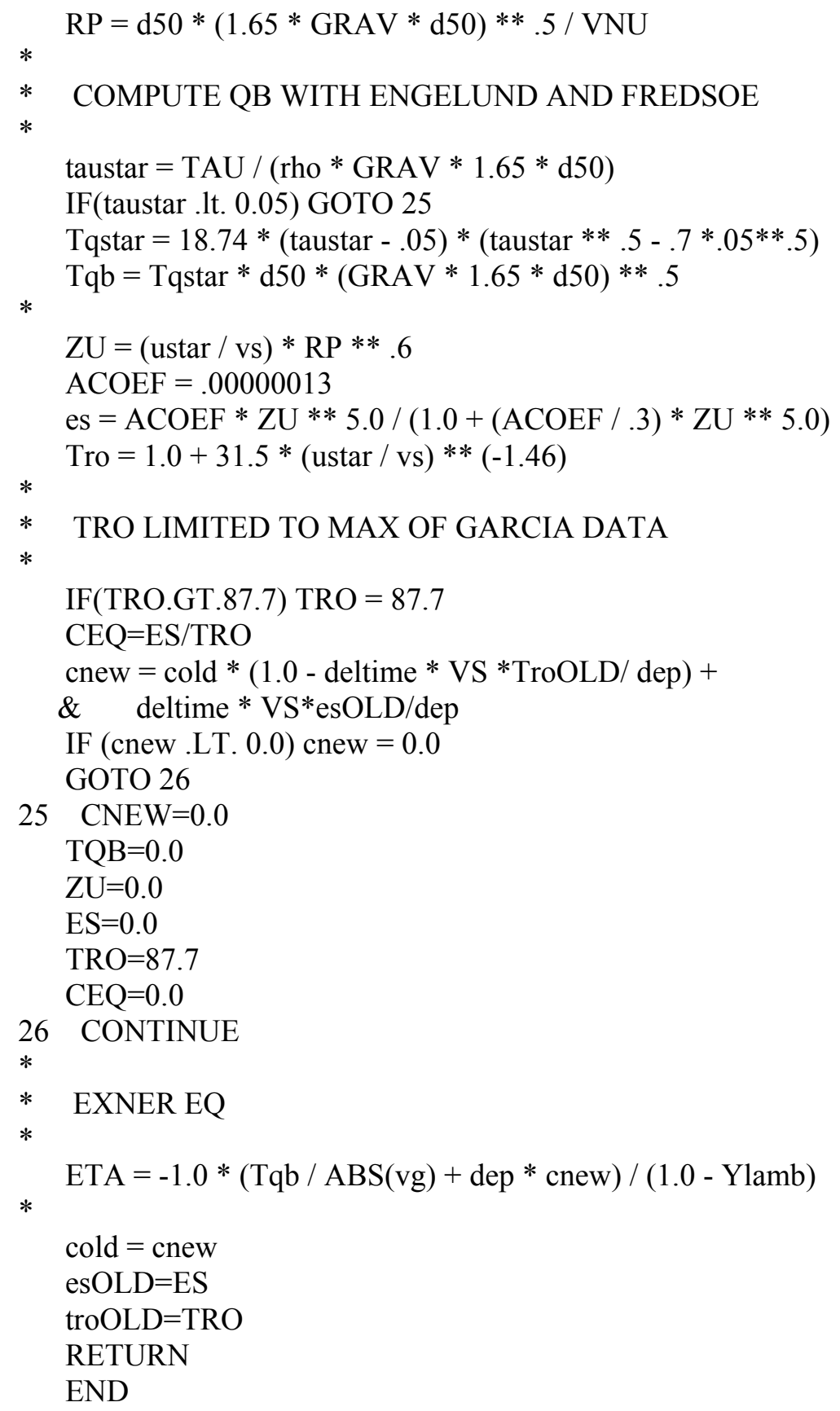




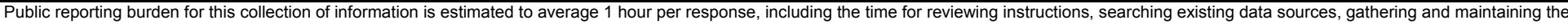

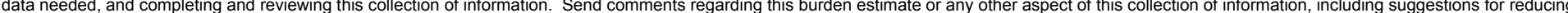

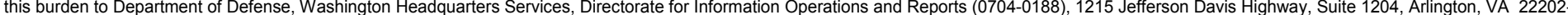

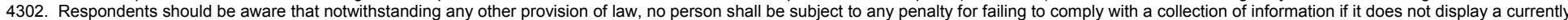
valid OMB control number. PLEASE DO NOT RETURN YOUR FORM TO THE ABOVE ADDRESS.

\begin{tabular}{l|l|l} 
1. REPORT DATE (DD-MM-YYYY) & 2. REPORT TYPE & 3. DATES COVERED (FrOm - To)
\end{tabular}

September 2002

Final report

\section{TITLE AND SUBTITLE}

User's Manual for NAVEFF Navigation Effects Model

5a. CONTRACT NUMBER

b. GRANT NUMBER

\section{AUTHOR(S)}

Scott Bourne

5c. PROGRAM ELEMENT NUMBER

5d. PROJECT NUMBER

5e. TASK NUMBER

5f. WORK UNIT NUMBER

\section{PERFORMING ORGANIZATION NAME(S) AND ADDRESS(ES)}

U.S. Army Engineer Research and Development Center

Environmental Laboratory

8. PERFORMING ORGANIZATION REPORT NUMBER

3909 Halls Ferry Road

Vicksburg, MS 39180-6199

ERDC/EL TR-02-29

\section{SPONSORING / MONITORING AGENCY NAME(S) AND ADDRESS(ES)}

10. SPONSOR/MONITOR'S ACRONYM(S)

U.S. Army Engineer District, Rock Island

Rock Island, IL 61204-2004

11. SPONSOR/MONITOR'S REPORT NUMBER(S)

\section{DISTRIBUTION / AVAILABILITY STATEMENT}

Approved for public release; distribution is unlimited.

\section{SUPPLEMENTARY NOTES}

\section{ABSTRACT}

The analysis of system-wide impacts begins with the Navigation Effects (NAVEFF) model. This user guide provides a detailed description of the NAVEFF model installation and execution. The format of the NAVEFF input and output files is documented in this user's guide. The NAVEFF model is a one-dimensional model that estimates the physical forces generated across a cross section caused by shallow draft navigation of a tow boat. NAVEFF is run at each half mile cross section for pools 4, 8, 13, 26, and LaGrange and at 1 mile cross section in the remaining pools. Inputs for NAVEFF are 108 different tow boat combinations, three river stages, and three sailing line locations. Output from NAVEFF includes maximum velocity change, maximum drawdown, maximum wave height, maximum bed scour, and maximum bed shear stress. The output from NAVEFF is one of the primary inputs to the other system ecological models and the sedimentation model.

\section{SUBJECT TERMS}

NAVEFF

Navigation Effects Model

Upper Mississippi River System (UMRS)

User Manual

\begin{tabular}{|c|c|c|c|c|c|}
\hline \multicolumn{3}{|c|}{ 16. SECURITY CLASSIFICATION OF: } & \multirow{2}{*}{$\begin{array}{l}\text { 17. LIMITATION } \\
\text { OF ABSTRACT }\end{array}$} & \multirow{2}{*}{$\begin{array}{l}\text { 18. NUMBER } \\
\text { OF PAGES }\end{array}$} & 19a. NAME OF RESPONSIBLE PERSON \\
\hline $\begin{array}{l}\text { a. REPORT } \\
\text { UNCLASSIFIED }\end{array}$ & $\begin{array}{l}\text { b. ABSTRACT } \\
\text { UNCLASSIFIED }\end{array}$ & $\begin{array}{l}\text { c. THIS PAGE } \\
\text { UNCLASSIFIED }\end{array}$ & & & $\begin{array}{l}\text { 19b. TELEPHONE NUMBER (include area } \\
\text { code) }\end{array}$ \\
\hline
\end{tabular}

